\title{
Maximal efficiency of the collisional Penrose process with spinning particles
}

\author{
Kei-ichi Maeda, ${ }^{1, *}$ Kazumasa Okabayashi, ${ }^{1, \dagger}$ and Hirotada Okawa ${ }^{2,3, *}$ \\ ${ }^{1}$ Department of Physics, Waseda University, Shinjuku, Tokyo 169-8555, Japan \\ ${ }^{2}$ Yukawa Institute for Theoretical Physics, Kyoto University, Kyoto 606-8502, Japan \\ ${ }^{3}$ Advanced Research Institute for Science and Engineering, Waseda University, Tokyo 169-8555, Japan
}

(Received 25 April 2018; published 14 September 2018)

\begin{abstract}
We analyze the collisional Penrose process of spinning test particles in an extreme Kerr black hole. We consider that two particles plunge into the black hole from infinity and collide near the black hole. For the collision of two massive particles, if the spins of particles are $s_{1} \approx 0.01379 \mu M$ and $s_{2} \approx-0.2709 \mu M$, we obtain the maximal efficiency is about $\eta_{\max }=($ extracted energy $) /($ input energy $) \approx 15.01$, which is more than twice as large as the case of the collision of non-spinning particles $\left(\eta_{\max } \approx 6.32\right)$. We also evaluate the collision of a massless particle without spin and a massive particle with spin (Compton scattering), in which we find the maximal efficiency is $\eta_{\max } \approx 26.85$ when $s_{2} \approx-0.2709 \mu M$, which should be compared with $\eta_{\max } \approx 13.93$ for the nonspinning case.
\end{abstract}

DOI: 10.1103/PhysRevD.98.064027

\section{INTRODUCTION}

A black hole is the most strongly bound system. If we can extract energy from a black hole, it would be much more efficient than nuclear energy. However, because of the black hole area theorem [1], we cannot extract energy from a Schwarzschild black hole. For a rotating black hole, instead, Penrose suggested the use of the ergoregion of a rotating black hole to extract energy [2]. A particle can have negative energy in the ergoregion. Hence we suppose that a plunged particle in the ergoregion breaks up into two particles such that one particle has negative energy and falls into the black hole, while the other particle with positive energy, which is larger than the input energy, goes away to infinity. As a result, we can extract energy from a rotating black hole, which is called Penrose process.

It was pointed out that this Penrose process could play a key role in the energy emission mechanism of jets and/or xrays from astrophysical objects [3]. It has become one of the most interesting and important mechanisms in astrophysics as well as in general relativity. However, some earlier works [4-6] showed that the incident particle or the break-up particles must be relativistic, which implies that the Penrose process is rare in astrophysics and that this process cannot serve for astrophysical process.

A disintegration of a plunged particle may also not be practical for extraction of energy from a black hole. Hence two more plausible methods have been proposed: One is a superradiance, in which we use propagating waves instead of

\footnotetext{
*maeda@waseda.jp

bayashioka@gravity.phys.waseda.ac.jp

h.okawa@aoni.waseda.jp
}

a particle [7-11]. An impinging wave on a rotating black hole is amplified for some range of frequencies when it is scattered (see [12] for the recent progress). The other one is a collisional Penrose process, in which two particles plunge into a black hole and collide in the ergoregion instead of disintegration of a single particle [13]. One expects that it may give more efficient mechanism in astrophysical situations. Unfortunately, the efficiency of the energy extraction, which is the ratio of the extracted energy to the input energy, turns to be as modest as the original Penrose process [14].

Recently this process has again attracted much attention because Bañados, Silk, and West [15] showed that the center of mass energy of two particles can be arbitrarily large when the angular momentum of one incident particle is tuned and the collision occurs near the horizon of an extreme Kerr black hole. This is referred to the BSW effect. If the center-of-mass energy is enough large, new unknown particles could be created if any. It may reveal new physics. It could also play an important role in astrophysics.

There have been so far many studies on the BSW effect after their finding [16-31]. Since the interaction between a black hole spin and an angular momentum of the particle is essential for the Penrose process and the BSW effect, it may be interesting to discuss collision of spinning particles. As we will summarize in the text, the 4-momentum of a spinning particle is not always parallel to its 4-velocity, resulting in the possibility of violation of the timelike condition of the orbit. As a result, although the BSW effect by collision of spinning particles in non-rotating Schwarzschild spacetime can take place near the horizon, the motion of the spinning particles becomes superluminal before the collision point [31]. While, if the particle energy satisfies $E<\sqrt{3} \mu / 6$, with which such a particle cannot 
plunge from infinity, the timelike condition is preserved until the horizon [32]. Of course, we find the BSW effect for the collision of spinning particles in a rapidly rotating Kerr (or Kerr-Newman) black hole $[33,34]$.

We are also very curious about the efficiency of energy extraction from a black hole, which is defined by $\eta=$ (output energy)/(input energy). Even when the centerof-mass energy becomes arbitrarily large near the horizon, a resulting particle may not necessarily escape to infinity. Thus, it is also important to study how large is the efficiency of the energy extraction from a black hole.

When two massive particles collide near the horizon on the equatorial plane and are converted to massless particles (photons), Bejger et al. [35] showed numerically that the maximal efficiency is about 1.29. This result has been confirmed analytically by Harada, Nemoto, and Miyamoto [36]. However, as Schnittman showed numerically [37], the maximal efficiency becomes 13.92 when an outgoing finetuned massless particle collides with a massive particle near the horizon. Leiderschneider and Piran [38] then derived the maximal efficiency analytically for several possible processes. They analyzed not only the collision on the equatorial plane but also more general offplane orbits. They concluded that the maximal efficiency is $(2+\sqrt{3})^{2} \approx 13.93$, which is found in the case of the Compton scattering (collision of massless and massive particles) on the equatorial plane. The similar analytic approaches were performed in $[39,40]$. These results agree with the numerical result by Schnittman [37].

More efficient way of extracting the energy from a black hole, which is called the super-Penrose process, has been proposed in [41,42], but there is still an argument [38]. The essential problem is how to create the particles which cause the super-Penrose process. Zaslavskii [43] pointed out that it is difficult to prepare a suitable initial state only by preceding mechanical collisions.

One natural question may arise: How the efficiency of the collisional Penrose process will be enhanced when the particles are spinning? Recently this subject was discussed in [44]. However the timelike condition was not properly taken into account. The value of spin is too large for the orbit to be timelike. Here we will study the effect of the particle spin on the efficiency of energy extraction in detail. We consider the collision of two massive spinning particles and the Compton or inverse Compton scattering (collision of one massless and one massive particles). In Sec. II, we briefly review the equation of motion of a spinning particle in a Kerr black hole and provide the timelike condition of the orbit. In Sec. III, we study the collision of two spinning particles in an extreme Kerr geometry and analyze the maximal efficiency. We also discuss the collision of one spinning massive particle and one massless particle (the Compton and the inverse Compton scatterings). Section IV is devoted to concluding remarks. Throughout this paper, we use the geometrical units of $c=G=1$ and follow [45] for the notations.

\section{BASIC EQUATIONS}

\section{A. Equations of motion of a spinning particle}

We consider a spinning particle in Kerr geometry. The equations of motion of a spinning particle were first derived by Papapetrou [46] by the use of the pole-dipole approximation of an extended body, and then reformulated by Dixon [47-49]. The equations of motion are

$$
\begin{aligned}
\frac{D p^{\mu}}{d \tau} & =-\frac{1}{2} R^{\mu}{ }_{\nu \rho \sigma} v^{\nu} S^{\rho \sigma} \\
\frac{D S^{\mu \nu}}{d \tau} & =p^{\mu} v^{\nu}-p^{\nu} v^{\mu}
\end{aligned}
$$

where $p^{\mu}, v^{\mu}=d z^{\mu} / d \tau$, and $S^{\mu \nu}$ are the 4-momentum, the 4 -velocity and the spin tensor of the particle, respectively. $\tau$ is the proper time and $z^{\mu}(\tau)$ is the orbit of the particle. We need a set of supplementary conditions

$$
S^{\mu \nu} p_{\nu}=0,
$$

which fixes the center of mass of the particle.

Defining the particle mass $\mu(>0)$ by $\mu^{2}=-p^{\mu} p_{\mu}$, we also use a specific 4-momentum $u^{\mu}$, which is defined by

$$
u^{\mu}=\frac{p^{\mu}}{\mu} .
$$

The normalized magnitude of spin $s$ is defined by

$$
S^{\mu \nu} S_{\mu \nu}=2 \mu^{2} s^{2}
$$

We also normalize the affine parameter $\tau$ as

$$
u^{\mu} v_{\mu}=-1 \text {. }
$$

We then find the relation between the 4-velocity and the specific 4-momentum as

$$
v^{\mu}-u^{\mu}=\frac{S^{\mu \nu} R_{\nu \rho \sigma \lambda} u^{\rho} S^{\sigma \lambda}}{2\left(\mu^{2}+\frac{1}{4} R_{\alpha \beta \gamma \delta} S^{\alpha \beta} S^{\gamma \delta}\right)},
$$

which means that the 4-velocity $v^{\mu}$ and the 4-momentum $p^{\mu}$ are not always parallel.

\section{B. Conserved quantities}

If we have a Killing vector $\xi_{\mu}$ in a background geometry, we obtain the conserved quantity

$$
Q_{\xi}=p^{\mu} \xi_{\mu}+\frac{1}{2} S^{\mu \nu} \nabla_{\mu} \xi_{\nu} .
$$

In the Kerr geometry, there are two Killing vectors:

$$
\begin{aligned}
\xi_{\mu}^{(t)} & =-\left(\sqrt{\frac{\Delta}{\Sigma}} e_{\mu}^{(0)}+\frac{a \sin \theta}{\sqrt{\Sigma}} e_{\mu}^{(3)}\right) \\
\xi_{\mu}^{(\phi)} & =a \sqrt{\frac{\Delta}{\Sigma}} \sin ^{2} \theta e_{\mu}^{(0)}+\frac{\left(r^{2}+a^{2}\right) \sin \theta}{\sqrt{\Sigma}} e_{\mu}^{(3)},
\end{aligned}
$$


where

$$
\Delta=r^{2}-2 M r+a^{2} \quad \Sigma=r^{2}+a^{2} \cos ^{2} \theta,
$$

and the tetrad basis $e_{\mu}^{(a)}$ is defined by

$$
e_{\mu}{ }^{(a)}=\left(\begin{array}{cccc}
\sqrt{\frac{\Delta}{\Sigma}} & 0 & 0 & -a \sqrt{\frac{\Delta}{\Sigma}} \sin ^{2} \theta \\
0 & \sqrt{\frac{\Sigma}{\Delta}} & 0 & 0 \\
0 & 0 & \sqrt{\Sigma} & 0 \\
-\frac{a}{\sqrt{\Sigma}} \sin \theta & 0 & 0 & \frac{\left(r^{2}+a^{2}\right)}{\sqrt{\Sigma}} \sin \theta
\end{array}\right) .
$$

Hence there are two conserved quantities in Kerr geometry, which are the energy $E$ and the $z$ component of the total angular momentum $J$ given by

$$
\begin{aligned}
E:=-Q_{\xi^{(t)}}= & \sqrt{\frac{\Delta}{\Sigma}} p^{(0)}+\frac{a \sin \theta}{\sqrt{\Sigma}} p^{(3)}+\frac{M\left(r^{2}-a^{2} \cos ^{2} \theta\right)}{\Sigma^{2}} S^{(1)(0)}+\frac{2 M a r \cos \theta}{\Sigma^{2}} S^{(2)(3)} \\
J:=Q_{\xi^{(\phi)}}= & a \sin ^{2} \theta \sqrt{\frac{\Delta}{\Sigma}} p^{(0)}+\frac{\left(r^{2}+a^{2}\right) \sin \theta}{\sqrt{\Sigma}} p^{(3)}+\frac{a \sin ^{2} \theta}{\Sigma^{2}}\left[(r-M) \Sigma+2 M r^{2}\right] S^{(1)(0)} \\
& +\frac{a \sqrt{\Delta} \sin \theta \cos \theta}{\Sigma} S^{(2)(0)}+\frac{r \sqrt{\Delta} \sin \theta}{\Sigma} S^{(1)(3)}+\frac{\cos \theta}{\Sigma^{2}}\left[\left(r^{2}+a^{2}\right)^{2}-a^{2} \Delta \sin ^{2} \theta\right] S^{(2)(3)} .
\end{aligned}
$$

\section{Equations of motion in the equatorial plane}

We introduce a specific spin vector $s^{(a)}$ by

$$
s^{(a)}=-\frac{1}{2 \mu} \epsilon_{(b)(c)(d)}^{(a)} u^{(b)} S^{(c)(d)}
$$

which is inversed as

$$
S^{(a)(b)}=\mu \epsilon_{(c)(d)}^{(a)(b)} u^{(c)} s^{(d)},
$$

where $\epsilon_{(a)(b)(c)(d)}$ is the totally antisymmetric tensor with $\epsilon_{(0)(1)(2)(3)}=1$.

In what follows, we consider only the particle motion in the equatorial plane $(\theta=\pi / 2)$ [50]. From this constraint, we find that the spin direction is always perpendicular to the equatorial plane. Hence only one component of $s^{(a)}$ is nontrivial, i.e.,

$$
s^{(2)}=-s
$$

If $s>0$, the particle spin is parallel to the black hole rotation, while when $s<0$, it is antiparallel.

As a result, the spin tensor is described as

$$
S^{(0)(1)}=-s p^{(3)}, \quad S^{(0)(3)}=s p^{(1)}, \quad S^{(1)(3)}=s p^{(0)} .
$$

We then obtain the conserved quantities as

$$
\begin{aligned}
E & =\frac{\sqrt{\Delta}}{r} p^{(0)}+\frac{(a r+M s)}{r^{2}} p^{(3)} \\
J & =\frac{\sqrt{\Delta}}{r}(a+s) p^{(0)}+\frac{r\left(r^{2}+a^{2}\right)+a s(r+M)}{r^{2}} p^{(3)} .
\end{aligned}
$$

From those equations, we find

$$
\begin{aligned}
& u^{(0)}=\frac{\left[\left(r^{3}+a(a+s) r+a M s\right) E-(a r+M s) J\right]}{\mu r^{2} \sqrt{\Delta}\left(1-\frac{M s^{2}}{r^{3}}\right)} \\
& u^{(3)}=\frac{[J-(a+s) E]}{\mu r\left(1-\frac{M s^{2}}{r^{3}}\right)} .
\end{aligned}
$$

There exists the normalization condition $u_{\mu} u^{\mu}=-1$, i.e.,

$$
-\left(u^{(0)}\right)^{2}+\left(u^{(1)}\right)^{2}+\left(u^{(3)}\right)^{2}=-1 .
$$

Hence we have

$$
u^{(1)}=\sigma \sqrt{\left(u^{(0)}\right)^{2}-\left(u^{(3)}\right)^{2}-1},
$$

where $\sigma= \pm 1$ correspond to the outgoing and ingoing motions, respectively. 
The relation between the 4-velocity $v^{(a)}$ and the specific 4-momentum $u^{(a)}$ is given by

$$
v^{(0)}=\Lambda_{s}^{-1} u^{(0)}, \quad v^{(1)}=\Lambda_{s}^{-1} u^{(1)}, \quad v^{(3)}=\frac{\left(1+\frac{2 M s^{2}}{r^{3}}\right)}{\left(1-\frac{M s^{2}}{r^{3}}\right)} \Lambda_{s}^{-1} u^{(3)}
$$

where

$$
\Lambda_{s}=1-\frac{3 M s^{2} r[J-(a+s) E]^{2}}{\mu^{2} \Sigma_{s}^{3}} \text { with } \Sigma_{s}=r^{2}\left(1-\frac{M s^{2}}{r^{3}}\right)
$$

Hence we obtain

$$
\begin{aligned}
& \frac{d t}{d \tau}:=v^{0}=\frac{r^{2}+a^{2}}{r \sqrt{\Delta}} v^{(0)}+\frac{a}{r} v^{(3)}=\frac{1}{r \Lambda_{s}}\left(\frac{r^{2}+a^{2}}{\sqrt{\Delta}} u^{(0)}+a \frac{1+\frac{2 M s^{2}}{r^{3}}}{1-\frac{M s^{2}}{r^{3}}} u^{(3)}\right), \\
& \frac{d r}{d \tau}:=v^{1}=\frac{\sqrt{\Delta}}{r} v^{(1)}=\frac{\sqrt{\Delta}}{r \Lambda_{s}} u^{(1)}, \\
& \frac{d \phi}{d \tau}:=v^{3}=\frac{a}{r \sqrt{\Delta}} v^{(0)}+\frac{1}{r} v^{(3)}=\frac{1}{r \Lambda_{s}}\left(\frac{a}{\sqrt{\Delta}} u^{(0)}+\frac{1+\frac{2 M s^{2}}{r^{3}}}{1-\frac{M s^{2}}{r^{3}}} u^{(3)}\right),
\end{aligned}
$$

We finally obtain the equations of motion of the spinning particle as

$$
\begin{aligned}
& \Sigma_{s} \Lambda_{s} \mu \frac{d t}{d \tau}=\frac{\Sigma_{s} \mu}{r}\left(\frac{r^{2}+a^{2}}{\sqrt{\Delta}} u^{(0)}+a \frac{1+\frac{2 M s^{2}}{r^{3}}}{1-\frac{M s^{2}}{r^{3}}} u^{(3)}\right)=a\left(1+\frac{3 M s^{2}}{r \Sigma_{s}}\right)[J-(a+s) E]+\frac{r^{2}+a^{2}}{\Delta} P_{s} \\
& \Sigma_{s} \Lambda_{s} \mu \frac{d r}{d \tau}=\frac{\Sigma_{s} \mu \sqrt{\Delta}}{r} u^{(1)}=\sigma \sqrt{R_{s}} \\
& \Sigma_{s} \Lambda_{s} \mu \frac{d \phi}{d \tau}=\frac{\Sigma_{s} \mu}{r}\left(\frac{a}{\sqrt{\Delta}} u^{(0)}+\frac{1+\frac{2 M s^{2}}{r^{3}}}{1-\frac{M s^{2}}{r^{3}}} u^{(3)}\right)=\left(1+\frac{3 M s^{2}}{r \Sigma_{s}}\right)[J-(a+s) E]+\frac{a}{\Delta} P_{s}
\end{aligned}
$$

where

$$
\begin{aligned}
& P_{s}=\left[r^{2}+a^{2}+\frac{a s}{r}(r+M)\right] E-\left(a+\frac{M s}{r}\right) J \\
& R_{s}=P_{s}^{2}-\Delta\left[\frac{\mu^{2} \Sigma_{s}^{2}}{r^{2}}+[-(a+s) E+J]^{2}\right] .
\end{aligned}
$$

Note that

$$
u^{(1)}=\sigma \frac{r \sqrt{R_{s}}}{\mu \sqrt{\Delta} \Sigma_{s}} .
$$

Now we introduce the dimensionless variables as

$$
\tilde{E}=\frac{E}{\mu}, \quad \tilde{J}=\frac{J}{\mu M}, \quad \tilde{s}=\frac{s}{M}, \quad \tilde{t}=\frac{t}{M}, \quad \tilde{r}=\frac{r}{M}, \quad a_{*}=\frac{a}{M}, \quad \tilde{\tau}=\frac{\tau}{M},
$$

and

$$
\tilde{\Delta}=\tilde{r}^{2}-2 \tilde{r}+a_{*}^{2},
$$




$$
\begin{aligned}
& \tilde{\Sigma}_{s}=\frac{\Sigma_{s}}{M^{2}}=\tilde{r}^{2}\left(1-\frac{\tilde{s}^{2}}{\tilde{r}^{3}}\right), \\
& \tilde{P}_{s}=\frac{P_{s}}{\mu M^{2}}=\left[\tilde{r}^{2}+a_{*}^{2}+\frac{a_{*} \tilde{s}}{\tilde{r}}(\tilde{r}+1)\right] \tilde{E}-\left(a_{*}+\frac{\tilde{s}}{\tilde{r}}\right) \tilde{J}, \\
& \tilde{R}_{s}=\frac{R_{s}}{\mu^{2} M^{4}}=\tilde{P}_{s}^{2}-\tilde{\Delta}\left[\frac{\tilde{\Sigma}_{s}^{2}}{\tilde{r}^{2}}+\left[-\left(a_{*}+\tilde{s}\right) \tilde{E}+\tilde{J}\right]^{2}\right] .
\end{aligned}
$$

The equations of motion are then given by

$$
\begin{aligned}
& \tilde{\Sigma}_{s} \Lambda_{s} \frac{d \tilde{t}}{d \tilde{\tau}}=a_{*}\left(1+\frac{3 \tilde{s}^{2}}{\tilde{r} \tilde{\Sigma}_{s}}\right)\left[\tilde{J}-\left(a_{*}+\tilde{s}\right) \tilde{E}\right]+\frac{\tilde{r}^{2}+a_{*}^{2}}{\tilde{\Delta}} \tilde{P}_{s} \\
& \tilde{\Sigma}_{s} \Lambda_{s} \frac{d \tilde{r}}{d \tilde{\tau}}= \pm \sqrt{\tilde{R}_{s}} \\
& \tilde{\Sigma}_{s} \Lambda_{s} \frac{d \phi}{d \tilde{\tau}}=\left(1+\frac{3 \tilde{s}^{2}}{\tilde{r} \tilde{\Sigma}_{s}}\right)\left[\tilde{J}-\left(a_{*}+\tilde{s}\right) \tilde{E}\right]+\frac{a_{*}}{\tilde{\Delta}} \tilde{P}_{s} .
\end{aligned}
$$

\section{Constraints on the orbits}

In what follows, we drop the tilde just for brevity. In order to find an orbit to the horizon $r_{H}:=1+\sqrt{1-a_{*}^{2}}$, the radial function $R_{s}$ must be non-negative for $r \geq r_{H}$, which condition is reduced to be

$$
\begin{gathered}
\left\{\left[r^{3}+a_{*}\left(a_{*}+s-b\right) r+\left(a_{*}-b\right) s\right]^{2}\right. \\
\left.-r^{2} \Delta\left(a_{*}+s-b\right)^{2}\right\} E^{2} \geq \Delta \Sigma_{s}^{2},
\end{gathered}
$$

by introducing an "impact" parameter $b:=J / E$. There exists a critical value of the impact parameter $b_{\text {cr }}$, beyond which the orbit cannot reach the event horizon. The particle bounces off at the turning point $d r / d \tau=0$, which radius is larger than $r_{H}$.

The turning point for the critical orbit with $b=b_{\text {cr }}$ is found just at the horizon radius. From the condition such that $R_{S}\left(r_{H}\right)=0$, we find

$$
b_{\mathrm{cr}}=\frac{r_{H}^{3}+a_{*}\left(a_{*}+s\right) r_{H}+a_{*} s}{a_{*} r_{H}+s}=a_{*}+s+\frac{r_{H}^{3}-s^{2}}{a_{*} r_{H}+s} .
$$

Hence in order for the orbit to reach the horizon, the condition such that $b \leq b_{\mathrm{cr}}$ is required.

There exists one more important physical condition that the 4-velocity must be timelike, which is explicitly written as

$$
\begin{aligned}
v^{\mu} v_{\mu} & =-\left(v^{(0)}\right)^{2}+\left(v^{(1)}\right)^{2}+\left(v^{(3)}\right)^{2} \\
& =\frac{\left[(1-X)^{2}\left(-\left(u^{(0)}\right)^{2}+\left(u^{(1)}\right)^{2}\right)+(1+2 X)^{2}\left(u^{(3)}\right)^{2}\right]}{\left[1-X\left(1+3\left(u^{(3)}\right)^{2}\right)\right]^{2}} \\
& <0,
\end{aligned}
$$

where $X=\frac{s^{2}}{r^{3}}$. It gives

$$
(1-X)^{2}\left(-\left(u^{(0)}\right)^{2}+\left(u^{(1)}\right)^{2}\right)+(1+2 X)^{2}\left(u^{(3)}\right)^{2}<0 .
$$

Since $-\left(u^{(0)}\right)^{2}+\left(u^{(1)}\right)^{2}+\left(u^{(3)}\right)^{2}=-1$, this condition is reduced to be

$$
-(1-X)^{2}+3 X(2+X)\left(u^{(3)}\right)^{2}<0 .
$$

From

$$
u^{(3)}=\frac{X^{1 / 3}}{s^{2 / 3}(1-X)}\left[J-\left(a_{*}+s\right) E\right],
$$

we obtain the timelike condition of $v^{\mu}$ as

$$
\frac{(1-X)^{4}}{(2+X) X^{5 / 3}}>\frac{3\left[J-\left(a_{*}+s\right) E\right]^{2}}{s^{4 / 3}} .
$$

This condition must be satisfied outside of the event horizon, $r \geq r_{H}$. Note that this timelike condition is always satisfied for $s=0$.

Since $s^{2} \leq 1, X$ is always smaller than unity outside of the horizon, and the function on the left hand side in the inequality (2.2) is monotonic with respect to $X$, we find the above condition is reduced to be

$$
\frac{\left(1-X_{H}\right)^{4}}{\left(2+X_{H}\right) X_{H}^{5 / 3}}>\frac{3\left[J-\left(a_{*}+s\right) E\right]^{2}}{s^{4 / 3}},
$$

where $X_{H}:=s^{2} / r_{H}^{3}$.

By use of the impact parameter $b$, we find the above timelike condition for as

$$
E^{2}<\frac{s^{4 / 3}\left(1-X_{H}\right)^{4}}{3\left(b-a_{*}-s\right)^{2}\left(2+X_{H}\right) X_{H}^{5 / 3}},
$$

which gives a constraint on the particle energy $E$.

It is also regarded as a constraint on the impact parameter $b$ for given energy $E$, i.e.,

$$
a_{*}+s-\frac{F\left(s, r_{H}\right)}{E}<b<a_{*}+s+\frac{F\left(s, r_{H}\right)}{E}
$$

where

$$
F\left(s, r_{H}\right):=\frac{s^{2 / 3}\left(1-X_{H}\right)^{2}}{\sqrt{3\left(2+X_{H}\right)} X_{H}^{5 / 6}} .
$$

For the critical orbit with $J=J_{\text {cr }}$, it becomes

$$
E^{2}<\frac{s^{4 / 3}\left(1-X_{H}\right)^{4}}{3\left(b_{\mathrm{cr}}-a_{*}-s\right)^{2}\left(2+X_{H}\right) X_{H}^{5 / 3}} .
$$

In what follows, we mainly consider the extreme Kerr black hole $\left(a_{*}=1, r_{H}=1\right)$, especially when we discuss the collisional Penrose process in the next section. For the extreme black hole, we find $b_{\text {cr }}=2$, which does not depend on the spin $s$. 
If the particle is not critical, by setting $b=2(1+\zeta)$, the timelike condition (2.4) is rewritten as

$$
\begin{gathered}
-\frac{(1-s)}{2}-\frac{\left(1-s^{2}\right)^{2}}{2 E \sqrt{3 s^{2}\left(2+s^{2}\right)}}<\zeta \\
<-\frac{(1-s)}{2}+\frac{\left(1-s^{2}\right)^{2}}{2 E \sqrt{3 s^{2}\left(2+s^{2}\right)}}
\end{gathered}
$$

This gives a constraint on $\zeta$ (or the impact parameter $b=J / E)$.

While, for the critical particle with $b_{\mathrm{cr}}=2$, from (2.5) we have the timelike condition as

$$
E^{2}<\frac{(1-s)^{2}(1+s)^{4}}{3 s^{2}\left(2+s^{2}\right)} .
$$

If the particle plunges from infinity, $E \geq 1$, which gives the constraint on the spin $s$ as $s_{\min }<s<s_{\max }$, where $s_{\min }$ and $s_{\max }$ are the solution of the equation

$$
s^{6}+2 s^{5}-4 s^{4}-4 s^{3}-7 s^{2}+2 s+1=0,
$$

with the constraint $s^{2} \leq 1$. We find $s_{\min } \approx-0.2709$ and $s_{\max } \approx 0.4499$.

Equation (2.7) also gives the constraint on a spin $s$ for given particle energy $E$, which is shown in Fig. 1. This shows the high energy particle cannot reach the horizon if the spin is too large.

When we will discuss a collision in the next chapter, we find that the direction of the particle is important. Since we assume two particles plunge from infinity, those particles are ingoing. However, if $b>b_{\mathrm{cr}}$, a particle falling from

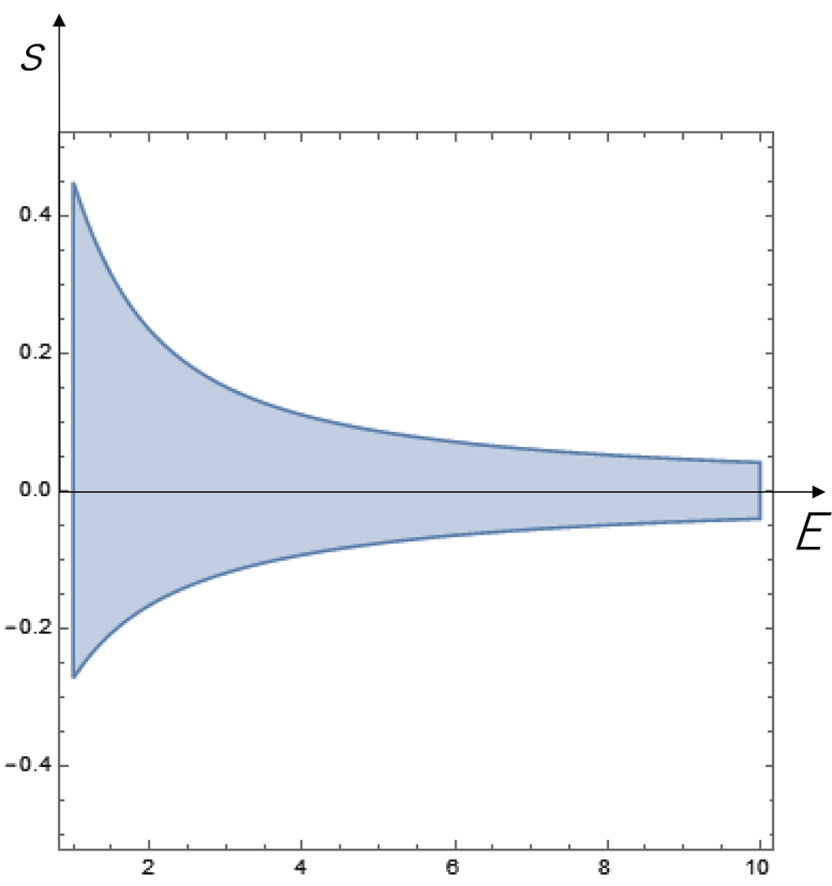

FIG. 1. The allowed region for the spin $s$ and the energy $E$, with which the particle can reach the event horizon.

infinity will find a turning point, and then bounce back to infinity. Such a particle is moving outward. Hence we consider both directions of the particle motions at collision.

Solving $d r / d \tau=0$ for the angular momentum $J$, we find $J=J_{ \pm}(r, E, \mu, s)$, where

$$
J_{ \pm}=\frac{E\left\{-2 r^{4}+r^{2}\left(r^{3}-3 r^{2}-2\right) s-r(r+1) s^{2}\right\} \pm(r-1)\left(r^{3}-s^{2}\right) \sqrt{E^{2} r^{4}-\mu^{2}\left(r^{2}+s\right)\left(r^{2}-2 r-s\right)}}{r\left(r^{2}+s\right)\left(r^{2}-2 r-s\right)}
$$

which gives the bounce point $r$ for a given value of $b=b_{ \pm}:=J_{ \pm} / E$.

Figure 2 shows the turning points for various values of the spin $s$ for $E=1$.

We find that $\min \left(b_{+}\right)=b_{\mathrm{cr}}$. Then, if the particle is near critical ( $b \approx b_{\text {cr }}$ ) but $b>b_{\text {cr }}$, the particle bounces back near the horizon.

For the negative value of $b$, when $b<\max \left(b_{-}\right)$, the outgoing particle near the horizon will bounce back to the horizon, while the particle coming from infinity will bounce back to infinity. We find $\max \left(b_{-}\right) \approx-4.97$, -4.82 , and -4.54 for $s=-0.27,0$, and 0.449 , respectively.

For nonextreme black hole, from Eq. (2.5), the timelike condition for the critical orbit with $E \geq 1$ gives the necessary conditions on the parameters of $\left(s, a_{*}\right)$, which is shown in Fig. 3. For $a_{*}=0.9, E \geq 1$ gives $-0.3179<s<0.5497$, which range is a little larger than the extreme case. While for $a_{*}=0$ (Schwarzschild black hole), no region exists because there is no critical orbit.

\section{E. Orbit of a massless particle on the equatorial plane}

Since we also discuss the scattering of massless particle later, we shall describe its orbit on the equatorial plane in the Kerr geometry. A massless particle is not spinning $(s=0)$. Hence, the conserved energy and the $z$-component of the angular momentum of the massless particle are defined by

$$
E=-p^{\mu} \xi_{\mu}^{(t)}, \quad \text { and } \quad J=p^{\mu} \xi_{\mu}^{(\phi)} .
$$

Then we find

$$
p^{(0)}=\frac{\left.\left[\left(r^{2}+a^{2}\right) E-a\right) J\right]}{r \sqrt{\Delta}}, \quad \text { and } \quad p^{(3)}=\frac{[J-a E]}{r} \text {. }
$$

This gives 


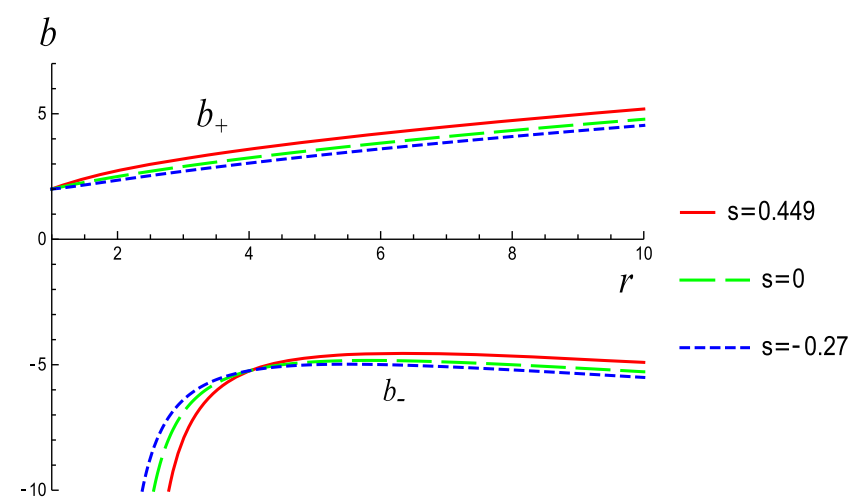

FIG. 2. The relation between the turning point $r$ and the impact parameter $b$ for a spinning particle with $E=1$. The particle with $b>b_{\text {cr }}$ or $b<\max \left(b_{-}\right)$falling from infinity will bounce at the turning point and escape to infinity, while the outward particle with $r<r_{\max }$ and $b<\max \left(b_{-}\right)$will bounce at the turning point and go back to the horizon, where $\max \left(b_{-}\right)=-4.97,-4.82$, and -4.54 and $r_{\max }=5.48,5.82$, and 6.30 for $s=-0.27$, 0 , and 0.449 , respectively.

$$
\begin{aligned}
p^{(1)} & =\sigma \sqrt{\left(p^{(0)}\right)^{2}-\left(p^{(3)}\right)^{2}} \\
& =\frac{\sigma}{r \sqrt{\Delta}} \sqrt{\left[\left(\left(r^{2}+a^{2}\right) E-a J\right)^{2}-(J-a E)^{2} \Delta\right]} .
\end{aligned}
$$

When we discuss the orbit we have to look at the 4-velocity $v^{\mu}=\frac{d z^{\mu}}{d \lambda}$, where $\lambda$ is an affine parameter. The 4-momentum $p^{\mu}$ and the 4-velocity $v^{\mu}$ are proportional. By choosing the affine parameter $\lambda$ appropriately, we can set

$$
p^{\mu}=E v^{\mu}
$$

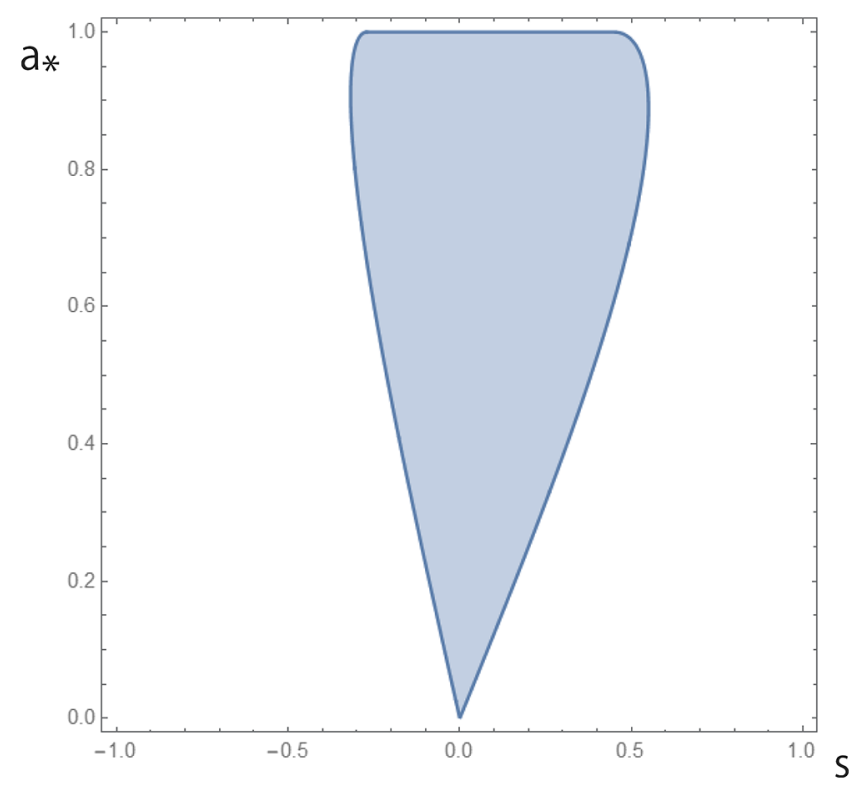

FIG. 3. The parameter region $\left(s, a_{*}\right)$ for the existence of the timelike critical orbit with $E \leq 1$ until the event horizon.
As a result, we find

$$
\begin{aligned}
\left(\frac{d r}{d \lambda}\right)^{2} & =\frac{\Delta}{r^{2}}\left(v^{(1)}\right)^{2}=\frac{\Delta}{r^{2}} \frac{\left(p^{(1)}\right)^{2}}{E^{2}} \\
& =\frac{1}{r^{4} E^{2}}\left[\left(\left(r^{2}+a^{2}\right) E-a J\right)^{2}-(J-a E)^{2} \Delta\right] .
\end{aligned}
$$

Using the "impact" parameter $b=J / E$, we find the critical value

$$
b_{\mathrm{cr}}=\frac{r_{H}^{2}+a^{2}}{a}=\frac{2 M r_{H}}{a}
$$

beyond which the photon orbit bounces before the horizon. For the extreme black hole, we find the same critical value $b_{\mathrm{cr}}=2$ as that for the massive particle.

\section{COLLISION OF SPINNING PARTICLES}

Now we discuss the collision of two particles moving in extreme Kerr geometry $\left(a_{*}=1\right)$, in which we expect the maximal energy extraction. Two particles 1 and 2, whose 4-momenta are $p_{1}^{\mu}$ and $p_{2}^{\mu}$, are moving to a rotating black hole and collide just before the horizon. After the collision, the particles 3 with the 4 -momentum $p_{3}^{\mu}$ is going away to infinity, while the particle 4 with the 4 -momentum $p_{4}^{\mu}$ falls into the black hole.

We assume that the sum of two momenta and spins, if any, are conserved at the collision, i.e.,

$$
\begin{aligned}
p_{1}^{\mu}+p_{2}^{\mu} & =p_{3}^{\mu}+p_{4}^{\mu} \\
S_{1}^{\mu \nu}+S_{2}^{\mu \nu} & =S_{3}^{\mu \nu}+S_{4}^{\mu \nu} .
\end{aligned}
$$

From those conservations with the Killing vectors, we find the conservations of the energy and total angular momentum,

$$
\begin{aligned}
E_{1}+E_{2} & =E_{3}+E_{4} \\
J_{1}+J_{2} & =J_{3}+J_{4} .
\end{aligned}
$$

We also obtain that the sum of the spins and the radial components of 4-momenta are conserved at the collision;

$$
\begin{aligned}
\mu_{1} s_{1}+\mu_{2} s_{2} & =\mu_{3} s_{3}+\mu_{4} s_{4} \\
p_{1}^{(1)}+p_{2}^{(1)} & =p_{3}^{(1)}+p_{4}^{(1)} .
\end{aligned}
$$

In what follows, we discuss two cases: (A) collision of two massive particles (MMM), and (B) collision of massless and massive particles; the Compton scattering (PMP) and inverse Compton scattering (MPM), where we use the symbols of MMM, PMP, and MPM following [38]. $\mathbf{P}$ and $\mathbf{M}$ describe a massless particle (a photon) and a massive particle, respectively. The first and the second 
letters denote colliding particles, while the third letter shows an escaped particle.

For the case (A) MMM, we assume all masses of the particles are the same, i.e., $\mu_{1}=\mu_{2}=\mu_{3}=\mu_{4}=\mu$. Hence the conservation equations hold for the dimensionless specific variables:

$$
\begin{gathered}
\tilde{E}_{1}+\tilde{E}_{2}=\tilde{E}_{3}+\tilde{E}_{4} \\
\tilde{J}_{1}+\tilde{J}_{2}=\tilde{J}_{3}+\tilde{J}_{4} \\
\tilde{s}_{1}+\tilde{s}_{2}=\tilde{s}_{3}+\tilde{s}_{4} \\
u_{1}^{(1)}+u_{2}^{(1)}=u_{3}^{(1)}+u_{4}^{(1)} .
\end{gathered}
$$

For the case (B) PMP, we assume that the particles 1 and 3 are massless and nonspinning, corresponding to a photon, while the particles 2 and 4 have the same mass, i.e., $\mu_{2}=\mu_{4}=\mu$. We then have

$$
\begin{gathered}
\tilde{s}_{2}=\tilde{s}_{4} \\
p_{1}^{(1)}+p_{2}^{(1)}=p_{3}^{(1)}+p_{4}^{(1)},
\end{gathered}
$$

in addition to two conservation equations (3.1) and (3.2). In the case of (B) MPM, the particles 2 and 4 are massless and nonspinning, while the particles 1 and 3 are massive with the same mass, i.e., $\mu_{1}=\mu_{3}=\mu$, and Eq. (3.5) is replaced by

$$
\tilde{s}_{1}=\tilde{s}_{3} .
$$

As we showed, there exists a critical orbit, which satisfies $J=J_{\text {cr }}=2 E$ in the extreme Kerr spacetime. This orbit will reach to the event horizon, and then bounce there. If $J<J_{\mathrm{cr}}$, the orbit gets into a black hole. While when $J>J_{\text {cr }}$, the orbit bounces back before the horizon.

We assume that the particles 1 and 2 starting from infinity are falling toward a black hole, and collide near the event horizon, i.e., the collision point $r_{c}$ is very close to the horizon $\left(r_{H}=1\right)$, i.e., $r_{c}=1 /(1-\epsilon)(0<\epsilon \ll 1)$. Hence the leading order of the radial component of the 4momentum $p^{(1)}$ is

$$
p^{(1)} \approx \sigma \frac{|2 E-J|}{\epsilon(1-s)}+\cdots .
$$

The momentum conservation equation $\left(p_{1}^{(1)}+p_{2}^{(1)}=\right.$ $\left.p_{3}^{(1)}+p_{4}^{(1)}\right)$ yields

$$
\begin{aligned}
\sigma_{1} & \frac{\left|2 E_{1}-J_{1}\right|}{1-s_{1}}+\sigma_{2} \frac{\left|2 E_{2}-J_{2}\right|}{1-s_{2}} \\
& =\sigma_{3} \frac{\left|2 E_{3}-J_{3}\right|}{1-s_{3}}+\sigma_{4} \frac{\left|2 E_{4}-J_{4}\right|}{1-s_{4}}+O(\epsilon)
\end{aligned}
$$

In what follows, we consider just the case such that the particle 1 is critical $\left(J_{1}=2 E_{1}\right)$.

To classify the case, we consider two situations for the particle orbits: One is near-critical $[J=2 E+O(\epsilon)]$, and the other is noncritical $\left[J=2 E+O\left(\epsilon^{0}\right)\right]$. Since we consider the collision near the horizon, non-critical orbit must have a smaller angular momentum $J<2 E$.

From Eq. (3.8), we find the following four cases:

(1) Both particle 2 and particle 3 are near-critical. In this case there is no constraint on $\sigma_{2}, \sigma_{3}$ and $\sigma_{4}$.

(2) The particle 2 is near-critical but the particle 3 is noncritical $\left(J_{3}<2 E_{3}\right)$. In this case, using the conservation equations (3.1) and (3.2), we find

$$
\left[\frac{\sigma_{3}}{1-s_{3}}+\frac{\sigma_{4}}{1-s_{4}}\right]\left(J_{3}-2 E_{3}\right)=O(\epsilon) .
$$

We find $\sigma_{4}=-\sigma_{3}$ and $s_{4}=s_{3}=s$. For the case (B), since $s_{3}=0$ or $s_{4}=0$, the massive particles are also nonspinning.

(3) The particle 3 is near-critical but the particle 2 is noncritical $\left(J_{2}<2 E_{2}\right)$. In this case, we find

$$
\left[\frac{\sigma_{4}}{1-s_{4}}-\frac{\sigma_{2}}{1-s_{2}}\right]\left(J_{2}-2 E_{2}\right)=O(\epsilon) .
$$

We find $\sigma_{4}=\sigma_{2}$ and $s_{4}=s_{2}$. Hence we have to impose $s_{3}=s_{1}$.

(4) Both particle 2 and particle 3 are noncritical $\left(J_{2}<2 E_{2}\right.$ and $\left.J_{3}<2 E_{3}\right)$. In this case there is no constraint on $\sigma_{2}, \sigma_{3}$ and $\sigma_{4}$.

Here we shall analyze only the case (3). It is because it gives a good efficiency as we will show below. We will not discuss the other three cases (1), (2), and (4) in this paper. It is because it does not seem to get a good efficiency for the cases (1) and (2). For the case (4), the super-Penrose process could be possible, but it is not possible to analyze it by our present method.

Since we consider the collision of the particle 1 and the particle 2, the noncritical particle 2 with $J_{2}<2 E_{2}$ must be ingoing $\left(\sigma_{2}=-1\right)$. So we assume that $\sigma_{4}=\sigma_{2}=-1$. While the critical particle 1 can be either ingoing $\left(\sigma_{1}=-1\right)$ or outgoing after a bounce near the horizon $\left(\sigma_{1}=1\right)$. The latter case is not exactly correct. In order for the particle 1 to bounce, it must be supercritical such that $J_{1}=2 E_{1}+\delta$ with $\delta>0$. We then take a limit of $\delta \rightarrow 0$, which gives the "critical orbit" with a bounce. Since we also have a small parameter $\epsilon$, we have to take a limit of $\delta \rightarrow 0$ first, which implies $\delta \ll \epsilon$. 
The above setting gives

From the conservation laws, we find

$$
\begin{gathered}
J_{1}=2 E_{1} \\
J_{3}=2 E_{3}\left(1+\alpha_{3} \epsilon+\beta_{3} \epsilon^{2}+\cdots\right),
\end{gathered}
$$

where $\alpha_{3}$ and $\beta_{3}$ are parameters of $O\left(\epsilon^{0}\right)$.

As for the particle 2, we assume

$$
J_{2}=2 E_{2}(1+\zeta)
$$

where $\zeta<0$ with $\zeta=O\left(\epsilon^{0}\right)$.

$$
E_{4}=E_{1}+E_{2}-E_{3}, \quad J_{4}=J_{1}+J_{2}-J_{3},
$$

giving

$$
J_{4}=2 E_{4}\left(1+\frac{E_{2}}{E_{4}} \zeta+\cdots\right)
$$

Now we evaluate $E_{2}$ and $E_{3}$ for the cases (A) and (B) separately.

\section{A. Case (A) MMM (Collision of two massive particles)}

For the massive particle, the radial component of the specific 4-momentum is written as

$$
u^{(1)}=\sigma \frac{r \sqrt{R_{s}}}{\Sigma_{s} \sqrt{\Delta}}=\frac{\sigma \sqrt{r^{2}\left[\left(r^{3}+(1+s) r+s\right) E-(r+s) J\right]^{2}-(r-1)^{2}\left[\left(r^{3}-s^{2}\right)^{2}+r^{4}(J-(1+s) E)^{2}\right]}}{(r-1)\left(r^{3}-s^{2}\right)} .
$$

Plugging the conditions (3.10) and (3.11) into Eq. (3.13), and using the conservation equations (3.12) we find

$$
\begin{gathered}
u_{1}^{(1)}=\sigma_{1}\left[\frac{f\left(s_{1}, E_{1}, 0\right)}{\left(1-s_{1}^{2}\right)}-\epsilon \frac{E_{1}^{2} h\left(s_{1}\right)}{\left(1-s_{1}^{2}\right)^{2} f\left(s_{1}, E_{1}, 0\right)}+O\left(\epsilon^{2}\right)\right] \\
u_{2}^{(1)}=\epsilon^{-1} \frac{2 E_{2}\left(1+s_{2}\right) \zeta}{1-s_{2}^{2}}-\frac{E_{2}\left(2+s_{2}\right)\left(1-s_{2}+2 \zeta\right)}{\left(1-s_{2}\right)^{2}\left(1+s_{2}\right)} \\
-\epsilon \frac{\left(1-s_{2}\right)^{4}\left(1+s_{2}\right)^{2}+E_{2}^{2}\left(1-s_{2}+2 \zeta\right)\left[\left(1-s_{2}\right)^{3}-2\left(1+2 s_{2}\right)\left(1+4 s_{2}+s_{2}^{2}\right) \zeta\right]}{4\left(1-s_{2}\right)^{3}\left(1+s_{2}\right)^{2} E_{2} \zeta}+O\left(\epsilon^{2}\right) \\
u_{3}^{(1)}=\sigma_{3}\left\{\frac{f\left(s_{1}, E_{3}, \alpha_{3}\right)}{\left(1-s_{1}^{2}\right)}-\left[\frac{\epsilon E_{3}^{2}}{\left(1-s_{1}^{2}\right)^{2} f\left(s_{1}, E_{3}, \alpha_{3}\right)} \times\left(h\left(s_{1}\right)-2\left(1+s_{1}\right)^{2}\left(2+s_{1}\right) g_{2}\left(s_{1}, \alpha_{3}\right)\right.\right.\right. \\
\left.\left.\left.\quad+2 \beta_{3}\left(1+s_{1}\right)\left(1-s_{1}^{2}\right) g_{1}\left(s_{1}, \alpha_{3}\right)\right)\right]+O\left(\epsilon^{2}\right)\right\} \\
u_{4}^{(1)}=\epsilon^{-1} \frac{2 E_{2}\left(1+s_{2}\right) \zeta}{1-s_{2}^{2}}-\frac{\left[E_{1}\left(1-s_{2}\right)\left(2+s_{2}\right)-E_{3}\left(1-s_{2}\right) g_{1}\left(s_{2}, \alpha_{3}\right)+E_{2}\left(2+s_{2}\right)\left(1-s_{2}+2 \zeta\right)\right]}{\left(1-s_{2}\right)^{2}\left(1+s_{2}\right)} \\
-\frac{\epsilon}{4\left(1-s_{2}\right)^{3}\left(1+s_{2}\right)^{2} E_{2} \zeta}\left[\left(1-s_{2}\right)^{4}\left[\left(E_{1}-E_{3}\right)^{2}+\left(1+s_{2}\right)^{2}\right]\right. \\
-2 E_{2}\left(1-s_{2}\right)\left\{4\left(1+s_{2}\right) E_{3} \zeta\left[\alpha_{3}\left(2+s_{2}\right)-\beta_{3}\left(1-s_{2}^{2}\right)\right]+\left(E_{3}-E_{1}\right)\left[\left(1-s_{2}\right)^{3}-2 s_{2}\left(2+s_{2}\right)^{2} \zeta\right]\right\} \\
\left.+E_{2}^{2}\left(1-s_{2}+2 \zeta\right)\left[\left(1-s_{2}\right)^{3}-2\left(1+2 s_{2}\right)\left(1+4 s_{2}+s_{2}^{2}\right) \zeta\right]\right]+O\left(\epsilon^{2}\right),
\end{gathered}
$$

where

$$
\begin{aligned}
f(s, E, \alpha) & :=\sqrt{E^{2}[3-2 \alpha(1+s)][1+2 s-2 \alpha(1+s)]-\left(1-s^{2}\right)^{2}}, \\
g_{1}(s, \alpha) & :=2+s-2 \alpha(1+s), \\
g_{2}(s, \alpha) & :=\alpha(2+s-2 \alpha) \\
h(s) & :=1+7 s+9 s^{2}+11 s^{3}-s^{4}
\end{aligned}
$$


Since $u_{1}^{(1)}+u_{2}^{(1)}=u_{3}^{(1)}+u_{4}^{(1)}$, we find the leading order of $\epsilon^{-1}$ is trivial. From the next leading order of $\epsilon^{0}$, we find

$$
\sigma_{3} \frac{f\left(s_{1}, E_{3}, \alpha_{3}\right)}{1-s_{1}^{2}}=\sigma_{1} \frac{f\left(s_{1}, E_{1}, 0\right)}{1-s_{1}^{2}}+\frac{\left[E_{1}\left(2+s_{2}\right)-E_{3} g_{1}\left(s_{2}, \alpha_{3}\right)\right]}{1-s_{2}^{2}}
$$

which is reduced to

$$
\mathcal{A} E_{3}^{2}-2 \mathcal{B} E_{3}+\mathcal{C}=0
$$

where

$$
\begin{gathered}
\mathcal{A}=-\left[3-2 \alpha_{3}\left(1+s_{1}\right)\right]\left[1+2 s_{1}-2 \alpha_{3}\left(1+s_{1}\right)\right]+\frac{\left(1-s_{1}^{2}\right)^{2}}{\left(1-s_{2}^{2}\right)^{2}} g_{1}^{2}\left(s_{2}, \alpha_{3}\right) \\
\mathcal{B}=g_{1}\left(s_{2}, \alpha_{3}\right) \frac{\left(1-s_{1}^{2}\right)}{\left(1-s_{2}^{2}\right)}\left[\left(2+s_{2}\right) \frac{\left(1-s_{1}^{2}\right)}{\left(1-s_{2}^{2}\right)} E_{1}+\sigma_{1} f\left(s_{1}, E_{1}, 0\right)\right] \\
\mathcal{C}=E_{1}\left[\left(\frac{3\left(1+2 s_{1}\right)\left(1-s_{2}^{2}\right)^{2}+\left(1-s_{1}^{2}\right)^{2}\left(2+s_{2}\right)^{2}}{\left(1-s_{2}^{2}\right)^{2}}\right) E_{1}+2 \sigma_{1} \frac{\left(1-s_{1}^{2}\right)\left(2+s_{2}\right)}{\left(1-s_{2}^{2}\right)} f\left(s_{1}, E_{1}, 0\right)\right],
\end{gathered}
$$

with the condition such that $E_{3} \leq E_{3, \text { cr }}$ for $\sigma_{3}=1$, or $E_{3} \geq E_{3, \text { cr }}$ for $\sigma_{3}=-1$, where

$$
E_{3, \mathrm{cr}}:=\frac{1}{g_{1}\left(s_{2}, \alpha_{3}\right)}\left[\left(2+s_{2}\right) E_{1}+\sigma_{1} \frac{\left(1-s_{2}^{2}\right)}{\left(1-s_{1}^{2}\right)} f\left(s_{1}, E_{1}, 0\right)\right]
$$

Here we focus just into the case of $\sigma_{3}=-1$. We should stress that for the outgoing particle 3 after collision $\left(\sigma_{3}=1\right)$, the energy $E_{3}$ has the upper bound $E_{3, \mathrm{cr}}$, which magnitude is the order of $E_{1}$. Hence we may not expect large efficiency. We will present the concrete analysis for the case of $\sigma_{3}=1$ in Appendix, in which we confirm the efficiency is not so high.

Since the particle 3 is ingoing after the collision, the orbit must be super-critical, i.e., $J_{3}>2 E_{3}$, which means either $\alpha_{3}>0$ or $\alpha_{3}=0$ with $\beta_{3}>0$. Once we give $\alpha_{3}$, the value of $E_{3}$ is fixed in terms of $s_{1}, s_{2}$ and $E_{1}$ by

$$
E_{3}=E_{3,+}:=\frac{\mathcal{B}+\sqrt{\mathcal{B}^{2}-\mathcal{A C}}}{\mathcal{A}}
$$

where we have chosen the larger root because it gives the larger extracted energy as it turns out that $\mathcal{A}$ is always positive.

The next leading order terms give

$$
\mathcal{P} E_{2}=\left(1-s_{2}\right)^{3}\left(E_{1}-E_{3}\right)^{2},
$$

where

$$
\begin{aligned}
\mathcal{P}:= & 2\left(E_{3}-E_{1}\right)\left(1-s_{2}\right)^{3}+4 \zeta\left[\frac{\left(1-s_{2}^{2}\right)^{2}}{\left(1-s_{1}^{2}\right)^{2}} \mathcal{Q}\right. \\
& +2\left(1+s_{2}\right) E_{3}\left[\alpha_{3}\left(2+s_{2}\right)-\beta_{3}\left(1-s_{2}^{2}\right)\right] \\
& \left.-s_{2}\left(2+s_{2}\right)^{2}\left(E_{3}-E_{1}\right)\right]
\end{aligned}
$$

with

$$
\begin{aligned}
\mathcal{Q}:= & \sigma_{1} \frac{E_{1}^{2} h\left(s_{1}\right)}{f\left(s_{1}, E_{1}, 0\right)}-\sigma_{3}\left[\frac{E_{3}^{2}}{f\left(s_{1}, E_{3}, \alpha_{3}\right)}\right. \\
& \times\left(h\left(s_{1}\right)-2\left(1+s_{1}\right)^{2}\left(2+s_{1}\right) g_{2}\left(s_{1}, \alpha_{3}\right)\right. \\
& \left.\left.+2 \beta_{3}\left(1+s_{1}\right)\left(1-s_{1}^{2}\right) g_{1}\left(s_{1}, \alpha_{3}\right)\right)\right]
\end{aligned}
$$

Since this fixes the value of $E_{2}$, we obtain the efficiency by

$$
\eta=\frac{E_{3}}{E_{1}+E_{2}}
$$

when $\alpha_{3}, \beta_{3}$ and $\zeta$ are given. 


\section{B. Case (B)}

\section{1. (B) PMP (Compton scattering)}

For the massless particle, we normalize the 4-momentum, the energy and the angular momentum by the mass $\mu$ of the massive particle. The radial component of the normalized 4-momentum is written as

$p^{(1)}=\frac{\sigma \sqrt{r[(r+1) E-J]\left[\left(r^{2}-r+2\right) E+(r-2) J\right]}}{r(r-1)}$,

where $E$ and $J$ are normalized by $\mu$ and $\mu M$ just as those of the massive particle.

For the momenta of the massive particles 2 and 4, Eqs. (3.15) and (3.17) do not change, while for the massless particles 1 and 3, we find

$$
\begin{gathered}
p_{1}^{(1)}=\sigma_{1}\left[\sqrt{3} E_{1}-\epsilon \frac{E_{1}}{\sqrt{3}}+O\left(\epsilon^{2}\right)\right] \\
p_{3}^{(1)}=\sigma_{3}\left\{E_{3} \sqrt{\left(3-\alpha_{3}\right)\left(1-2 \alpha_{3}\right)}\right. \\
\left.-\epsilon E_{3}\left[\frac{\left[1-4\left(2 \alpha_{3}-\beta_{3}\right)\left(1-\alpha_{3}\right)\right]}{\sqrt{\left(3-2 \alpha_{3}\right)\left(1-2 \alpha_{3}\right)}}\right]+O\left(\epsilon^{2}\right)\right\} .
\end{gathered}
$$

From the conservation of the radial components of the 4-momenta, we find

$$
E_{3}=\mathcal{S} E_{1},
$$

where the magnification factor $\mathcal{S}$ is given by

$$
\mathcal{S}:=\frac{\sigma_{1} \sqrt{3}\left(1-s_{2}^{2}\right)+2+s_{2}}{\sigma_{3} \sqrt{\left(3-2 \alpha_{3}\right)\left(1-2 \alpha_{3}\right)}\left(1-s_{2}^{2}\right)+2+s_{2}-2 \alpha_{3}\left(1+s_{2}\right)}
$$

and

$$
\mathcal{P} E_{2}=\left(1-s_{2}\right)^{3}\left(E_{1}-E_{3}\right)^{2},
$$

where $\mathcal{P}$ is given by Eq. (3.24) with $s_{1}=0$ but replacing $\mathcal{Q}$ by $\mathcal{T}$, which is defined by

$$
\mathcal{T}:=\sigma_{1} \frac{E_{1}}{\sqrt{3}}-\sigma_{3} E_{3}\left[\frac{1-4\left(2 \alpha_{3}-\beta_{3}\right)\left(1-\alpha_{3}\right)}{\sqrt{\left(3-\alpha_{3}\right)\left(1-2 \alpha_{3}\right)}}\right] .
$$

\section{Case (B) MPM (Inverse Compton scattering)}

For the momenta of the massive particles 1 and 3, Eqs. (3.14) and (3.16) do not change, while for the massless particles 2 and 4 , we find

$$
p_{2}^{(1)}=2 \epsilon^{-1} E_{2} \zeta-2 E_{2}(1+2 \zeta)-\epsilon \frac{E_{2}\left(1-4 \zeta^{2}\right)}{4 \zeta}+O\left(\epsilon^{2}\right)
$$

$$
\begin{aligned}
p_{4}^{(1)}= & 2 \epsilon^{-1} E_{2} \zeta-2\left[E_{4}+2 E_{2} \zeta+E_{3} \alpha_{3}\right] \\
& -\epsilon \frac{E_{4}^{2}-8 E_{2} E_{3}\left(2 \alpha_{3}-\beta_{3}\right) \zeta-4 E_{2}^{2} \zeta^{2}}{4 E_{2} \zeta}+O\left(\epsilon^{2}\right) .
\end{aligned}
$$

where $E_{4}=E_{1}+E_{2}-E_{3}$

From the conservation of the radial components of the 4momenta, we find

$$
E_{3}=\left.\frac{\mathcal{B}+\sqrt{\mathcal{B}^{2}-\mathcal{A C}}}{\mathcal{A}}\right|_{s_{2}=0},
$$

and

$$
E_{2}=\left.\frac{\left(E_{1}-E_{3}\right)^{2}}{\mathcal{P}}\right|_{s_{2}=0},
$$

where $\mathcal{A}, \mathcal{B}, \mathcal{C}$ and $\mathcal{P}$ are given by Eqs. (3.19)-(3.21), and (3.24), which should be evaluated with $s_{2}=0$. As a result, $E_{2}$ and $E_{3}$ coincide with those found at the collision of a spinning massive particle and a nonspinning massive particle.

\section{THE MAXIMAL EFFICIENCY}

\section{A. Efficiency of collision of massive particles}

Now we discuss the necessary condition to find the maximal efficiency. As we showed, giving the particle 1 energy $\left(E_{1}\right)$ and two particle spins $\left(s_{1}\right.$ and $\left.s_{2}\right)$, we find the energies of the particle 3 and particle 2 in terms of the orbit parameters of the particles 2 and $3\left(\alpha_{3}, \beta_{3}\right.$ and $\left.\zeta\right)$. In order to get the large efficiency, we must find large extraction energy, i.e., the energy of the particle $3\left(E_{3}\right)$ for given values of $E_{1}$ and $E_{2}$ of the ingoing particles. Although $E_{1}$ is arbitrary, the energy of the particle $2\left(E_{2}\right)$ is fixed in our approach. Hence we also have to find the possible minimum value of $E_{2}$. Since we consider two particles are plunging from infinity, we have the constraints of $E_{1} \geq 1$ and $E_{2} \geq 1$.

We then assume that $E_{1}=1$ and $\sigma_{1}=1$, and find the maximal value of $E_{3}$ as well as the minimum value of $E_{2}$. Note that we do not find a good efficiency for $\sigma_{1}=-1$, although the off-plane orbits may give a little better efficiency [38].

First we analyze $E_{3}$, which is determined by Eq. (3.22) for given value of $\alpha_{3}$. Since the orbit of the particle 3 is near critical, we have two constraints: $E_{3} \geq E_{3, \text { cr }}$ for $\sigma_{3}=-1$ and the timelike condition (2.7).

In order to find the large value of $E_{3}$, from the timelike condition we find that the spin magnitude $s_{3}\left(=s_{1}\right)$ must be small (see Fig. 1). Hence we first set $s_{1}=0$. We then show 


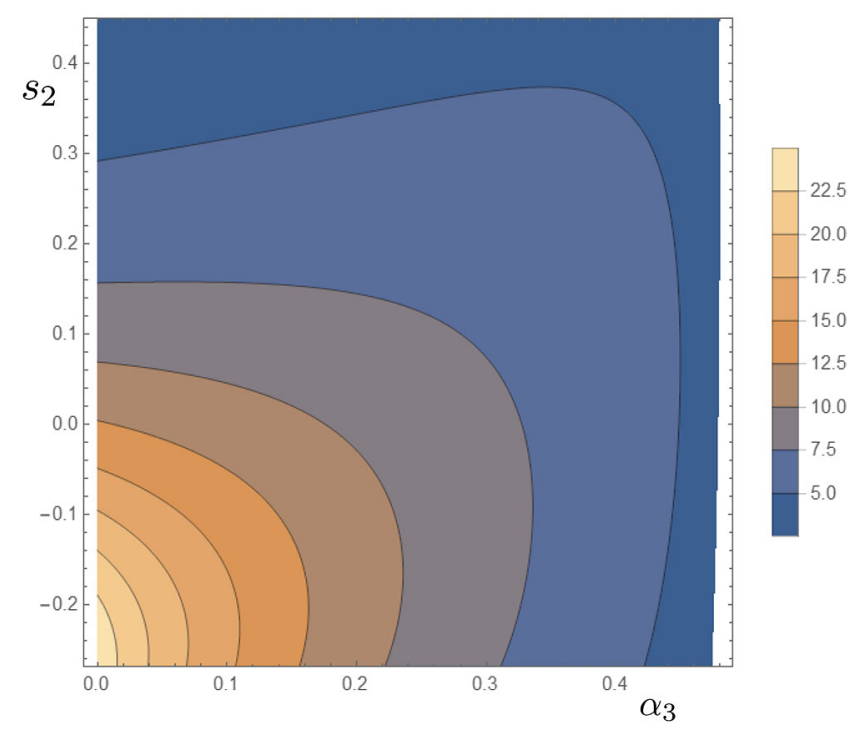

FIG. 4. The contour map of $E_{3}$ in terms of $\alpha_{3}$ and $s_{2}$ with $s_{1}=0 . E_{3}$ changes smoothly with respect to two parameters $\alpha_{3}$ and $s_{2}$, and $\alpha_{3} \rightarrow 0$ and small $s_{2}$ give larger value of $E_{3}$.

the contour map of $E_{3}$ in terms of $\alpha_{3}$ and $s_{2}$ in Fig. 4. We find $\alpha_{3} \approx 0$ gives the largest efficiency. Hence next we set $\alpha_{3}=0+$, and analyze the maximal efficiency. Here $0+$ means that we assume $\alpha_{3}>0$ but take a limit of $\alpha_{3} \rightarrow 0$ after taking the limit of $\epsilon \rightarrow 0$. This is justified because $E_{2}$ and $E_{3}$ change smoothly when we take the limit of $\alpha_{3} \rightarrow 0$.

Assuming $\alpha_{3}=0+$, we look for the maximal value of $E_{3}$ for given $s_{1}$ and $s_{2}$. In Fig. 5, we show the contour map of $E_{3}$ in terms of $s_{1}$ and $s_{2}$. The red point, which is $\left(s_{1}, s_{2}\right) \approx\left(0.01379, s_{\min }\right)$, gives the maximal value of $E_{3}$.

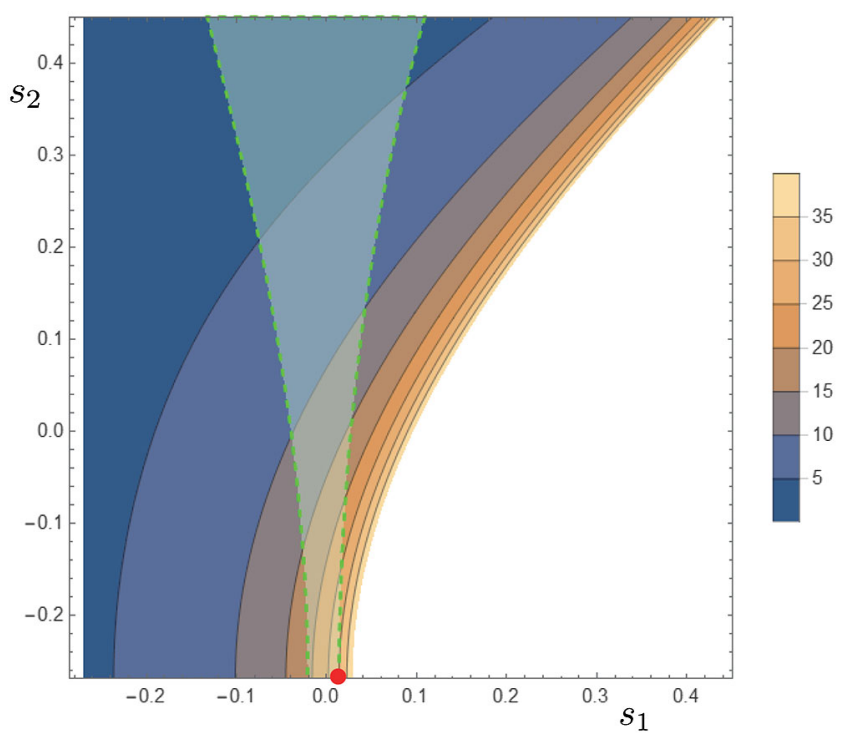

FIG. 5. The contour map of $E_{3}$ in terms of $s_{1}$ and $s_{2}$. The timelike condition for the particle 3 orbit is satisfied in the light green shaded region. As a result, the maximal value of $E_{3}=$ $E_{3, \text { max }} \approx 30.02$ is obtained when $s_{2}=s_{\text {min }} \approx-0.2709$ and $s_{1} \approx$ 0.01379 (the red point in the figure).
Since $E_{2} \geq 1$ when we plunge the particle 2 from infinity, if $E_{2}=1$ is possible, we find that the maximal value of $E_{3}$ gives the maximal efficiency. However $E_{2}$ is fixed in our approach. So we have to check whether $E_{2}=1$ is possible or not and then provide which conditions are required if possible.

The condition for $E_{2}=1$ in Eq. (3.23) gives the relation between $\zeta$ and $\beta_{3}$, which is a linear equation of $\beta_{3}$. Hence we always find a real solution of $\beta_{3}$. While the timelike condition of the particle 2 gives the constraint on $\zeta$, which is Eq. (2.6) with $E=1$, i.e.,

$$
\zeta_{\min }<\zeta<0
$$

where

$$
\zeta_{\min }:=-\frac{\left(1-s_{2}\right)}{2}\left[1+\frac{\left(1-s_{2}\right)\left(1+s_{2}\right)^{2}}{\sqrt{3 s_{2}^{2}\left(2+s_{2}^{2}\right)}}\right]
$$

since the upper bound in Eq. (2.6) is always positive for the range of $s_{\min }<s_{2}<s_{\max }$. For the parameters giving the maximal value of $E_{3}$, we find the relation between $\zeta$ and $\beta_{3}$, which is shown in Fig. 6. From the timelike condition for the particle 2 orbit, we have the constraint of $\zeta_{\min }<\zeta<0$ where $\zeta_{\min } \approx-1.271$.

Since there exists a possible range of parameters with $E_{2}=1$, we find the maximal efficiency is given by $\eta_{\max }=$ $E_{3, \max } / 2 \approx 15.01$.

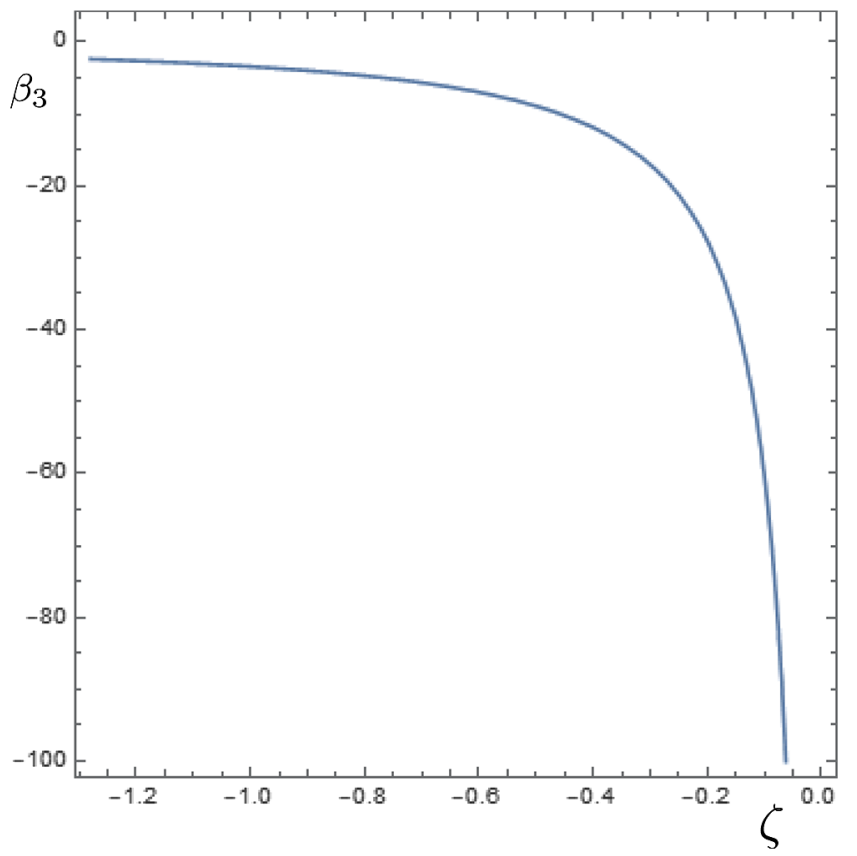

FIG. 6. The relation between $\zeta$ and $\beta_{3}$ for $E_{2}=1$. The other parameters are chosen to give the maximal value of $E_{3}$. The timelike condition for the particle 2 orbit gives the constraint of $\zeta_{\min }<\zeta<0$ with $\zeta_{\min } \approx-1.271$. 


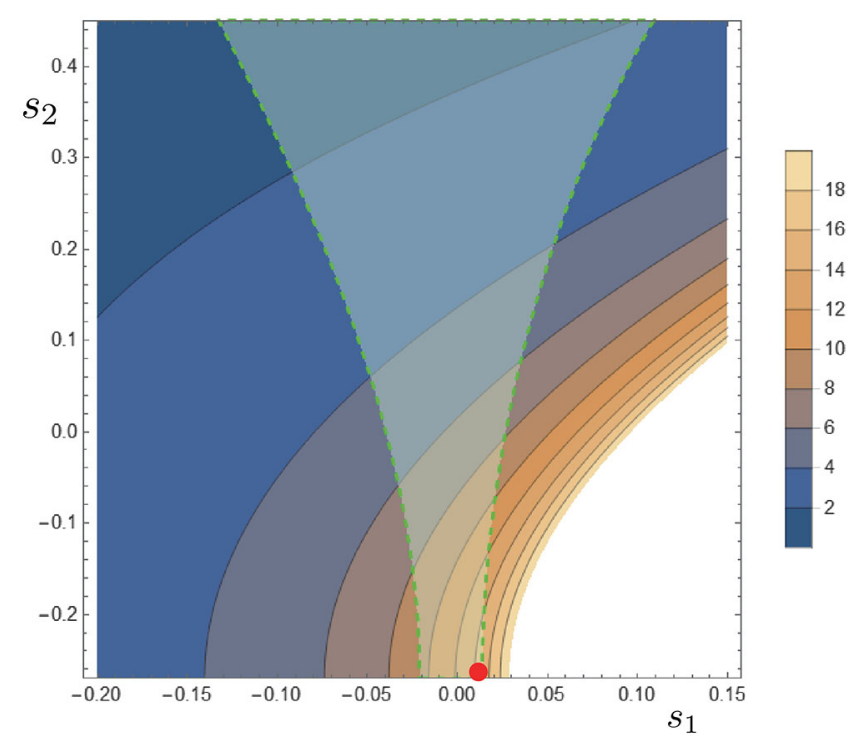

FIG. 7. The contour map of the maximal efficiency for given $s_{1}$ and $s_{2}$. The green shaded region is the constraint from the timelike condition of the particle 3 . The red point, $\left(s_{1}, s_{2}\right) \approx(0.01379$, $\left.s_{\text {min }}\right)$, gives the maximal efficiency $\eta_{\max } \approx 15.01$.

Hence we find the maximal efficiency $\eta_{\max }=E_{3} / 2$ for given $s_{1}$ and $s_{2}$, which is shown in Fig. 7. We also show the efficiency in terms of $s_{2}$ for fixed values of $s_{1}=-2.111 \times 10^{-2}, 0$ and $1.379 \times 10^{-2}$ in Fig. 8. The efficiency gets larger as $s_{2}$ approaches the minimum value $s_{\min }$. It shows that the effect of spin is very important. Note that we obtain the maximal efficiency $\eta_{\max } \approx 6.328$ for non-spinning case, which is consistent with [38].

\section{B. Efficiency of Compton scattering}

We find the efficiency $\eta$ by

$$
\eta=\frac{E_{3}}{E_{1}+E_{2}}=\frac{\mathcal{S}}{1+\frac{(\mathcal{S}-1)^{2}\left(1-s_{2}\right)^{3}}{\mathcal{P} / E_{1}}}
$$

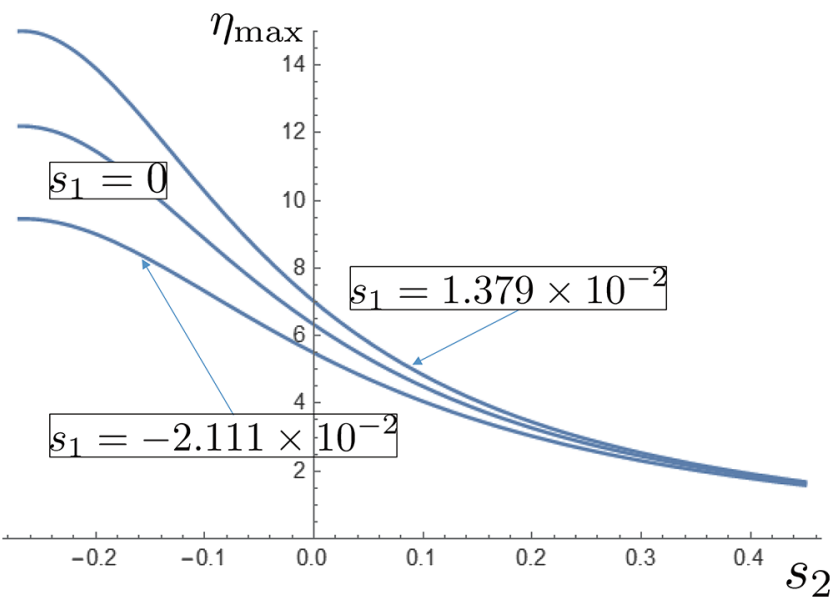

FIG. 8. The efficiency in terms of $s_{2}$ for fixed values of $s_{1}=-2.111 \times 10^{-2}, 0$ and $1.379 \times 10^{-2}$. where

$$
\begin{aligned}
\mathcal{P} / E_{1}= & 2(\mathcal{S}-1)\left(1-s_{2}\right)^{3} \\
& +4 \zeta\left[\left(1-s_{2}^{2}\right)^{2} \mathcal{T} / E_{1}+2\left(1+s_{2}\right) \mathcal{S}\left[\alpha_{3}\left(2+s_{2}\right)\right.\right. \\
& \left.\left.-\beta_{3}\left(1-s_{2}^{2}\right)\right]-s_{2}\left(2+s_{2}\right)^{2}(\mathcal{S}-1)\right]
\end{aligned}
$$

with

$$
\mathcal{T} / E_{1}=\frac{\sigma_{1}}{\sqrt{3}}-\sigma_{3} \mathcal{S}\left[\frac{1-4\left(2 \alpha_{3}-\beta_{3}\right)\left(1-\alpha_{3}\right)}{\sqrt{\left(3-\alpha_{3}\right)\left(1-2 \alpha_{3}\right)}}\right] .
$$

Although the extracted photon energy depends on the input photon energy $E_{1}$, the efficiency does not depend on $E_{1}$ and $E_{2}$. It is determined by the orbital parameters $\alpha_{3}, \beta_{3}$, and $\zeta$ as well as the spin $s_{2}$.

We first look for when we find the largest value of $E_{3}$, or the magnification factor $\mathcal{S}$, which is determined by $\alpha_{3}$. In Fig. 9, we show the magnification factor $\mathcal{S}$ in terms of $\alpha_{3}$ and $s_{2}$. Just as the case (A), $\alpha_{3} \rightarrow 0$ and small $s_{2}$ give larger value of $\mathcal{S}$. The maximal value is $\mathcal{S}_{\max } \approx 26.85$ at $\alpha_{3}=0+$ and $s_{2}=s_{\min } \approx-0.2709$.

Since the maximal value of $\mathcal{S}$ is obtained when $\alpha_{3} \rightarrow 0$ and $s_{2}=s_{\min }$, setting $\alpha_{3}=0+$ and $s_{2}=s_{\min }$, we show the contour map of the efficiency $\eta$ in terms of $\beta_{3}$ and $\zeta$ in Fig. 10.

Although $\beta_{3}$ is arbitrary as long as $\alpha_{3}>0, \zeta$ is constrained as $\zeta_{\min }<\zeta<0$ in order for the particle 2 can reach the horizon, where the minimum value $\zeta_{\min }$ depends on the spin $s_{2}$. For $s_{2}=s_{\min }$, we find $\zeta_{\min }=-3.890$. We then obtain the maximum efficiency for the Comptom scattering as $\eta_{\max }=26.85$ in the limit of $\beta_{3} \rightarrow-\infty$. If $s_{2}=0$, the maximal efficiency is $\eta_{\max } \approx 13.93$, which is consistent

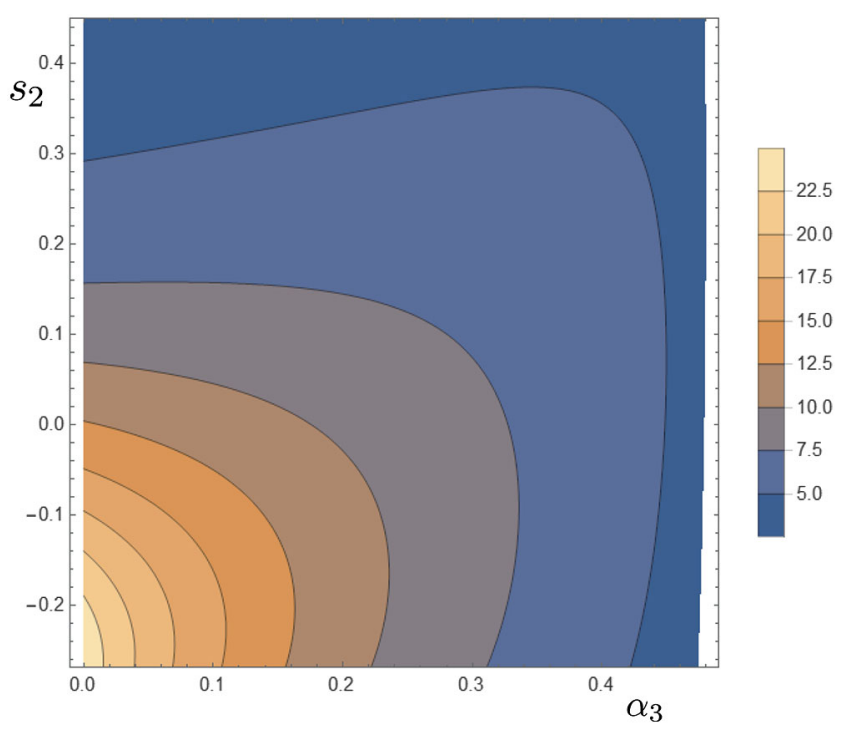

FIG. 9. The contour map of $\mathcal{S}$ in terms of $\alpha_{3}$ and $s_{2} . \mathcal{S}$ changes smoothly with respect to two parameters $\alpha_{3}$ and $s_{2}$, and $\alpha_{3} \rightarrow 0$ and small $s_{2}$ give larger value of $\mathcal{S}$. 


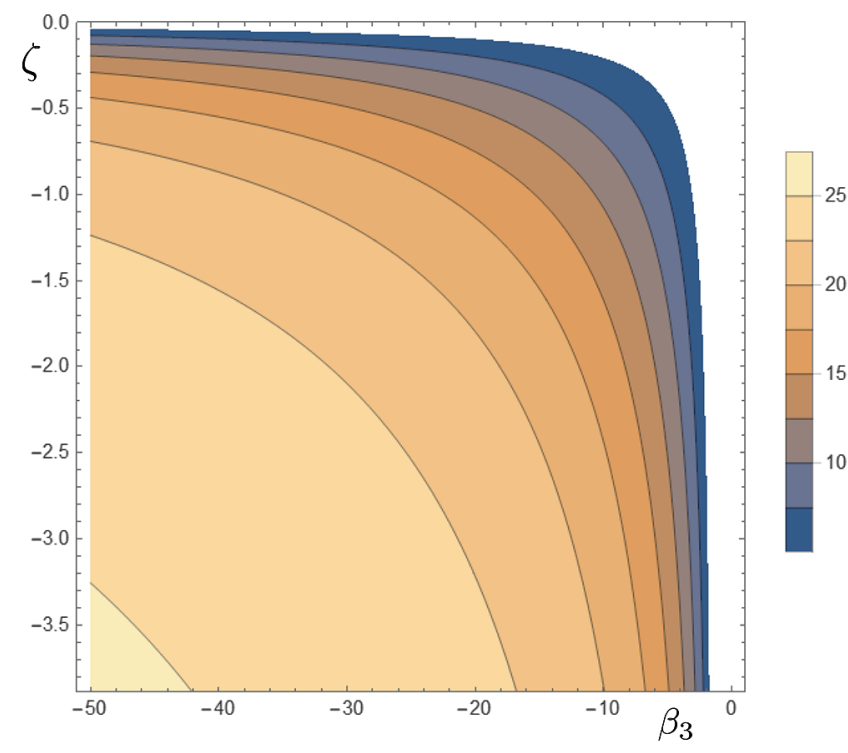

FIG. 10. The contour map of the efficiency in terms of $\beta_{3}$ and $\zeta$. Fixing $\zeta$ with $0>\zeta>\zeta_{\min }(\approx-3.890)$, in the limit of $\beta_{3} \rightarrow \infty$, we find the maximal efficiency of $\eta_{\max } \approx 26.85$.

with the results by Schnittman [37] and LeiderschneiderPiran [38].

\section{Efficiency of inverse Compton scattering}

Since the particles 1 and 2 plunge from infinity, we have the constraint $E_{1} \geq 1$ and $E_{2} \geq 0$. We then assume that $E_{1}=1$, and find the maximal value of $E_{3}$ as well as the minimal value of $E_{2}$. Since $E_{3}$ is determined only by $\alpha_{3}$ and $s_{1}$, we first discuss $E_{3}$.

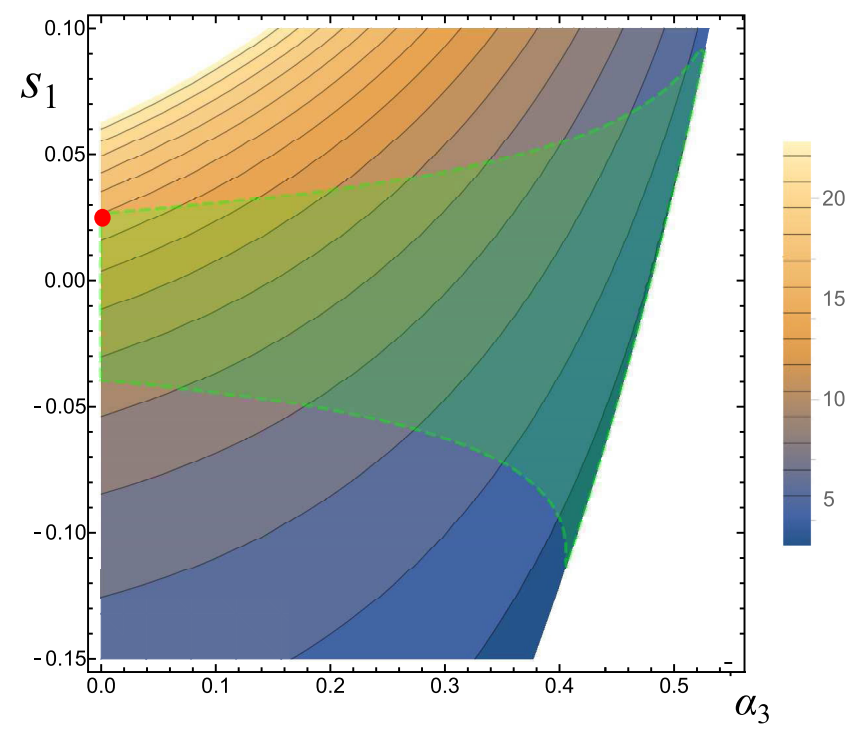

FIG. 11. The contour map of $E_{3}$ in terms of $\alpha_{3}$ and $s_{1}$. The timelike condition for the particle 3 is satisfied in the light-green shaded region. The maximum value of $E_{3}=E_{3, \max }=15.64$ is obtained at the red point $\left(\alpha_{3}, s_{1}\right)=(0,0.02679)$.
In Fig. 11, we show the contour map of $E_{3}$ in terms of $\alpha_{3}$ and $s_{1}$. The red point, which is $\left(\alpha_{3}, s_{1}\right)=(0,0.02679)$, gives the maximal value of $E_{3}$.

If $E_{2} \rightarrow 0$ is possible, it gives the minimal value of $E_{2}$ and then the maximal efficiency is given by $\eta_{\max }=E_{3, \max }$. Hence, assuming $\alpha_{3}=0+$ and $s_{1}=0.02679$, we analyze whether $E_{2} \rightarrow 0$ is possible or not. From Eq. (3.24), we find the asymptotic behavior of $\mathcal{P}$ as

$$
\mathcal{P} \approx 8 E_{3} \zeta \beta_{3}\left[\frac{E_{3}\left(2+s_{1}\right)}{\left(1-s_{1}\right) f\left(s_{1}, E_{3}, 0\right)}-1\right]
$$

if $\zeta \beta_{3} \rightarrow \infty$. It gives $E_{2} \rightarrow 0$. $\zeta$ is constrained as $-\infty<$ $\zeta<0$ because the particle 2 is nonspinning, while $\beta_{3}$ is arbitrary as long as $\alpha_{3}>0$. As a result, we obtain $E_{2} \rightarrow 0$ is obtained in the limit of $\zeta \beta_{3} \rightarrow \infty$. $\beta_{3}$ must be negative. Hence, we find the maximum efficiency $\eta_{\max } \approx 15.64$ for the inverse Compton scattering. For $s_{1}=0$, the maximum efficiency becomes $\eta_{\max }=7+4 \sqrt{2} \approx 12.66$, which is consistent with the result by Leiderschneider and Piran [38].

\section{CONCLUDING REMARK}

We have analyzed the maximal efficiency of the energy extraction from the extreme Kerr black hole by collisional Penrose process of spinning test particles. We summarize our result in Table I.

For the collision of two massive particles (MMM+), we obtain the maximal efficiency is about $\eta_{\max } \approx 15.01$, which is more than twice as large as the case of the collision of non-spinning particles. It happens when the particle 1 with $E_{1}=\mu, J_{1}=2 \mu M$ and $s_{1} \approx 0.01379 \mu M$ and the particle 2 with $E_{2}=\mu,-0.5418 \mu M<J_{2}<2 \mu M$ and $s_{2}=$ $s_{\text {min }} \approx 0.2709 \mu M$ plunge from infinity, and collide near the horizon. After collision, the particle 3 with $E_{3} \approx$ $30.02 \mu$ and $J_{3} \approx 60.03 \mu M$ escapes into infinity, while the particle 4 with $E_{4} \approx-28.02 \mu$ and $-58.57 \mu M<J_{4}<$ $-56.03 \mu M$ falls into the black hole.

As for the collision of a massless and massive particles, we obtain the maximal efficiency $\eta_{\max } \approx 26.85$ for the case of $\mathbf{P M P}+$ (the Compton scattering), which is almost twice as large as the nonspinning case. In the case of MPM+(the inverse Compton scattering), however, we find $\eta_{\max } \approx 15.64$, which value is not so much larger than the non-spinning case. It is because that the timelike condition forces the magnitude of spin not to be so large for the energetic spinning particle.

Although we have presented some examples to give a large efficiency of the energy extraction from a rotating black hole, the following cases should also be studied:

(1) Nonextreme black hole.-The spin of the astrophysical black hole may not exceed $a / M=0.998$ as pointed out by Thorne [51]. Hence we should analyze the efficiency for a non-extreme black hole. 
TABLE I. The maximal efficiencies and energies for three processes. We include the nonspinning case obtained by [38] as a reference. The maximal efficiencies and maximal energies are enhanced twice or more when the spin effect is taken into account. Following [38], we use the symbols of $\mathbf{M M M}+, \mathbf{P M P}+, \mathbf{M P M}+$ for each process, where + means the case of $\sigma_{1}=1$.

\begin{tabular}{lcccc}
\hline \hline & Spin $\left(s_{1}, s_{2}\right)$ & $\begin{array}{c}\text { Input energy } \\
\left(E_{1}, E_{2}\right)\end{array}$ & $\begin{array}{c}\text { Output energy } \\
\left(E_{3}\right)\end{array}$ & $\begin{array}{c}\text { Maximal } \\
\text { efficiency }\end{array}$ \\
\hline $\begin{array}{l}\text { MMM+ } \\
\quad(\text { Collisional process }\end{array}$ & Non-spinning & $(\mu, \mu)$ & $12.66 \mu$ & 6.328 \\
$\mathbf{P M P}+$ & & $30.02 \mu$ & 15.01 \\
$\quad($ Compton Scattering) & Non-spinning & $(+\infty, \mu)$ & $+\infty$ & 13.93 \\
MPM+ & $(0,-0.2709 \mu M)$ & & $+\infty$ & 26.85 \\
$\quad($ Inverse Compton Scattering) & Non-spinning & $(\mu, 0)$ & $12.66 \mu$ & 12.66 \\
\hline \hline
\end{tabular}

(2) Super-Penrose process.-We have not analyzed the case (4) : Collision of two subcritical particles. If $\sigma_{1}=1$, which is not a natural initial condition for a subcritical particle, there is no upper bound for the efficiency [38]. This super-Penrose process may be interesting to study for spinning particles too, although there still exists the question about its initial set up [43]. Recently it was discussed in [52], but the timelike condition has not been taken into account.

(3) Spin transfer.-Since a spin plays an important role in the efficiency, it is also interesting to discuss a transfer of spins. For example, $s_{1}=s_{2}=s_{\min } \approx$ -0.27 to $s_{3}=0$ and $s_{4}=2 s_{\min } \approx-0.54$.

(4) Collision of particles in off-equatrial-plane orbits.In [38], they also analyzed the collision of the particle in off-plane orbits, which gives the maximal efficiency for the case of $\sigma_{1}=-1$. Although it may be interesting to analyze the orbits not in the equatorial plane, the equations of motion for a spinning particle are not integrable. As a result, such an analysis would be very difficult.

(5) Backreaction effect.-In this paper, we have adopted a test particle approximation. However because of lack of the back reaction, it may not reveal the proper upper bound on the efficiency of the energy extraction. In the Reissner-Nordström spacetime, we could perform such an analysis for the collision of charged shells [53]. However it would be difficult to analyze the back reaction effect in Kerr black hole background although it is important.

Finally one may ask how large the magnitude of spin can be in a realistic astrophysical system since we have assumed a theoretically (or logically) allowed value of a spin in this paper. The orbital angular momentum is given by $|\boldsymbol{L}|=|\boldsymbol{r} \times \boldsymbol{p}| \sim R_{\text {orbit }} \times \mu v \gtrsim O(\mu M)$, while the spin angular momentum is $s \sim R_{\text {body }} \times \mu v \gtrsim O\left(\mu^{2}\right)$. Hence the ratio $s / L \sim R_{\text {body }} / R_{\text {orbit }}$ should be small for a test particle approximation. In fact, if a test particle is a black hole $\left(s \leq \mu^{2}\right)$, we find $s / \mu M=s / \mu^{2} \times(\mu / M) \ll 1$. Hence the value assumed here may be too large for astrophysical objects. However, for a fast rotating star, $s$ can be much larger than $\mu^{2}$. For example, we find $s / \mu^{2} \lesssim 500$ for a fast rotator $\alpha$ LEONIS (REGULUS) [54]. Hence the validity of the test particle approximation would be marginal in this case. The present spin effect might become important when we extend beyond a test particle limit including nonlinear or nonperturbed process.

\section{ACKNOWLEDGMENTS}

We would like to thank Tomohiro Harada and Kota Ogasawara for useful discussions. This work was supported in part by JSPS KAKENHI Grants No. JP16K05362 (KM) and No. JP17H06359 (KM).

\section{APPENDIX: THE CASE (3) WITH $\sigma_{3}=1$}

\section{Case (A)MMM (Collision of two massive particles)}

In this case, the condition $E_{3} \leq E_{3, \text { cr }}$ must be satisfied. As a result, $E_{3,+}$, which is the larger root of Eq. (3.18), is excluded. The possible solution is

$$
E_{3}=E_{3,-}:=\frac{\mathcal{B}-\sqrt{\mathcal{B}^{2}-\mathcal{A C}}}{\mathcal{A}} .
$$

$E_{3, \text { cr }}$ increases monotonically with respect to $\alpha_{3}$. $E_{3, \text { cr }}$ is positive for $\alpha_{3}<\alpha_{3, \infty}:=\frac{2+s_{2}}{2\left(1+s_{2}\right)}$, and $E_{3, \mathrm{cr}} \rightarrow \infty$ as $\alpha_{3} \rightarrow \alpha_{3, \infty}$, while beyond $\alpha_{3, \infty}, E_{3, \text { cr }}$ becomes negative, which case should be excluded. As $\alpha_{3}$ increases, $E_{3}$ also increases but faster than $E_{3, \text { cr }}$ and reaches the upper bound $E_{3, \mathrm{cr}}$ at some value of $\alpha_{3}=\alpha_{3, \mathrm{cr}}$.

For given values of $s_{1}$ and $s_{2}$, we find the quadratic equation for $\alpha_{3, \text { cr }}$ from the condition $E_{3, \text { cr }}=E_{3}$. Inserting the solution $\alpha_{3, \text { cr }}\left(s_{1}, s_{2}\right)$ into the definition of $E_{3}$, we find the largest value of $E_{3}, E_{3, \text { cr }}\left(s_{1}, s_{2}\right)$ in terms of $s_{1}$ and $s_{2}$. We show its contour map in Fig. 12, in which we also plot the timelike condition by the light-green shaded region. 


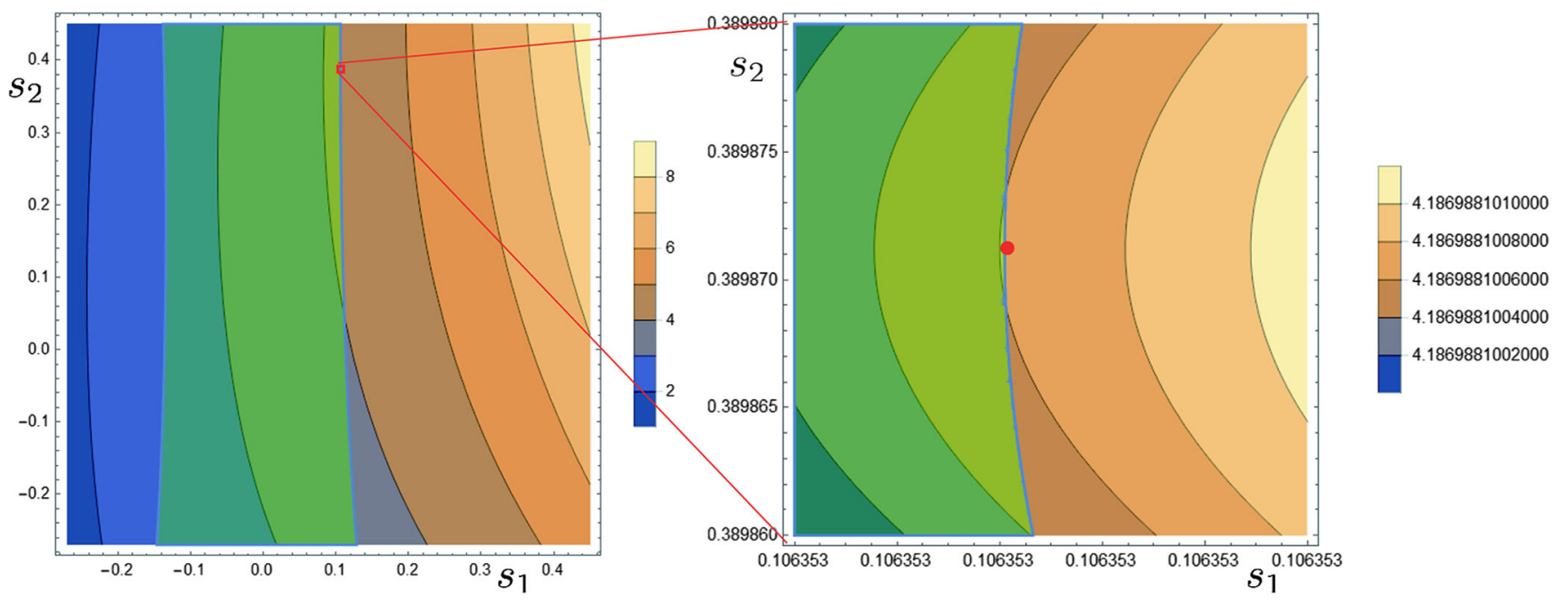

FIG. 12. The contour map of $E_{3, \text { cr }}\left(s_{1}, s_{2}\right)$ with $\alpha_{3}=\alpha_{3, \text { cr }}$, which gives the largest value of $E_{3}$ for given $s_{1}$ and $s_{2}$. The timelike condition for the particle 3 is satisfied in the light-green shaded region. The right figure is enlarged near the maximal point $\left(\left(s_{1}, s_{2}\right) \in(0.1063534539,0.1063534540) \times(0.38986,0.38988)\right)$. The maximal value $E_{3, \max } \approx 4.187$ is obtained at the red point $\left(s_{1}, s_{2}\right) \approx(0.10635,0.3899)$ with $\alpha_{3} \approx 0.5342$.

This gives the maximal value $E_{3, \max } \approx 4.187$ at the red point $\left(s_{1}, s_{2}\right) \approx(0.10635,0.3899,0.534)$ with $\alpha_{3} \approx 0.5342$.

We then check that $E_{2}=1$ is possible for the above parameters. Figure 13 shows the relation between $\zeta$ and $\beta_{3}$ for $E_{2}=1$. We conclude that the maximal efficiency is $\eta_{\max }=E_{3, \max } / 2 \approx 2.093$.

\section{Case (B) PMP Compton scattering}

In this case, since the particle 1 is massless, we first draw the contour map of the magnification factor $\mathrm{S}$, which is

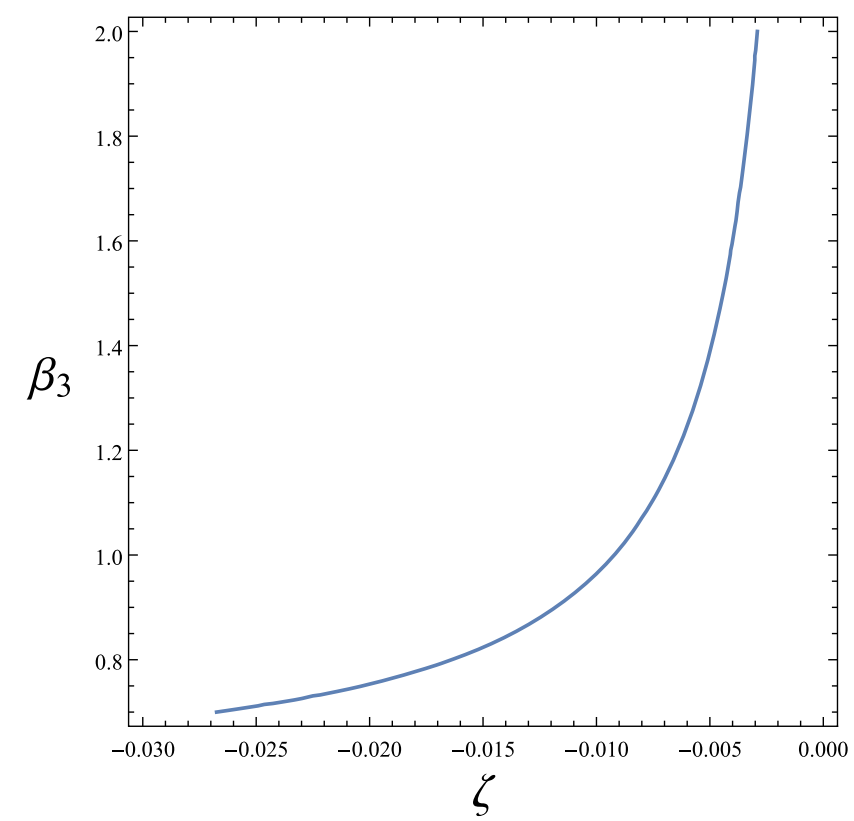

FIG. 13. The relation between $\zeta$ and $\beta_{3}$ for $E_{2}=1$ when $E_{3}$ takes the maximal value. defined by Eq. (3.29), in Fig. 14. The maximal value of $\mathcal{S}$ is $\mathcal{S}_{\max } \approx 3.876$, which is found at the red point $\left(\alpha_{3}, s_{2}\right) \approx(0.5,0.2887)$. We note that the value of $\alpha_{3}$ must be either $\alpha_{3} \leq 0.5$ or $\alpha_{3} \geq 1.5$ in order to find a real value of $\mathcal{S}$. However there is the constraint as $\alpha_{3} \leq 1$ from the future-directed proper time condition of $d t / d \lambda>0$, although the larger value of $\mathcal{S}$ is possible for $\alpha_{3}>1.5$. As a result, we find the above maximal value of $\mathcal{S}_{\max }$.

Figure 15 show that the efficiency is more than 3.85 in the wide range of parameters $\left(\zeta_{\min } \approx-3.326 \lesssim \zeta \lesssim-0.5\right.$ and $\beta_{3} \gtrsim 0.5$ ) and it takes the maximal value 3.854 , which

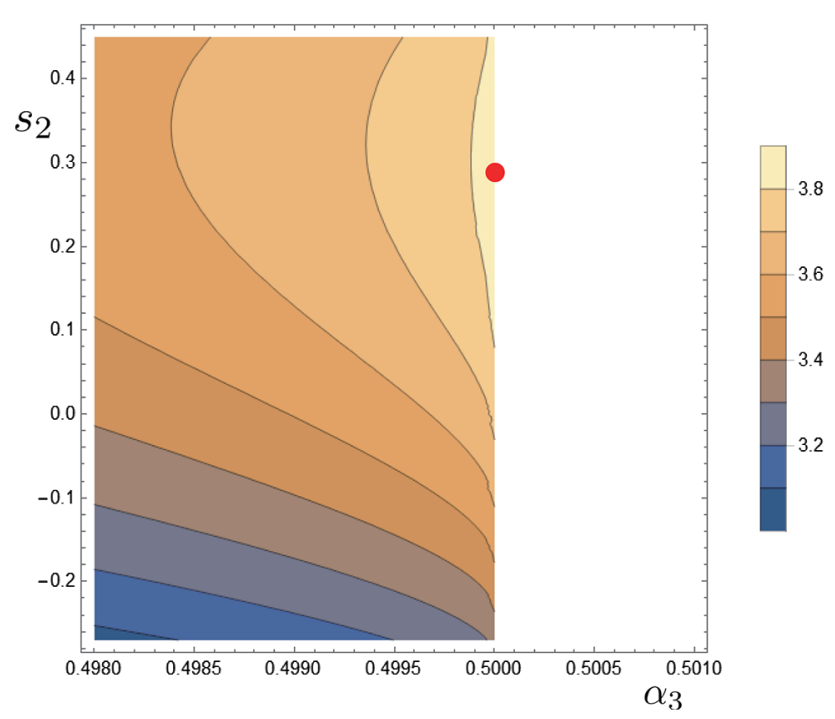

FIG. 14. The contour map of the magnification factor $\mathcal{S}$ in terms of $\alpha_{3}$ and $s_{2}$ for the Compton scattering in the case of $\sigma_{3}=1$. The maximal value $\mathcal{S}_{\max } \approx 3.876$ is found at the red point $\left(\alpha_{3}, s_{2}\right) \approx(0.5,0.2887)$. 


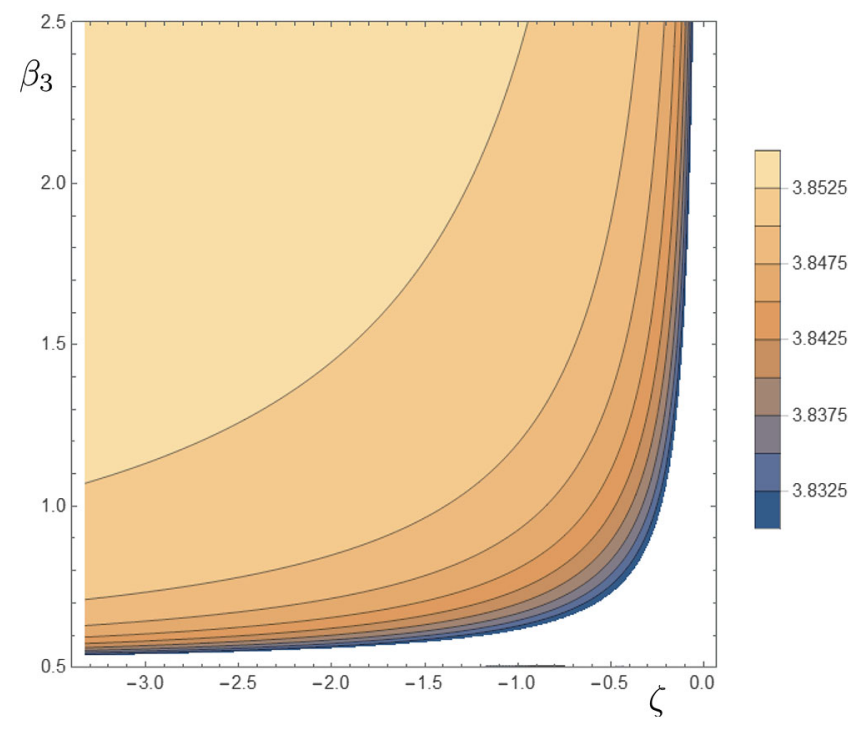

FIG. 15. The contour map of the efficiency $\eta$ in terms of $\zeta$ and $\beta_{3}$. The maximal value of the efficiency is about 3.853 in the limit of $\zeta \rightarrow \zeta_{\min } \approx-3.326$ and $\beta_{3} \rightarrow \infty$.

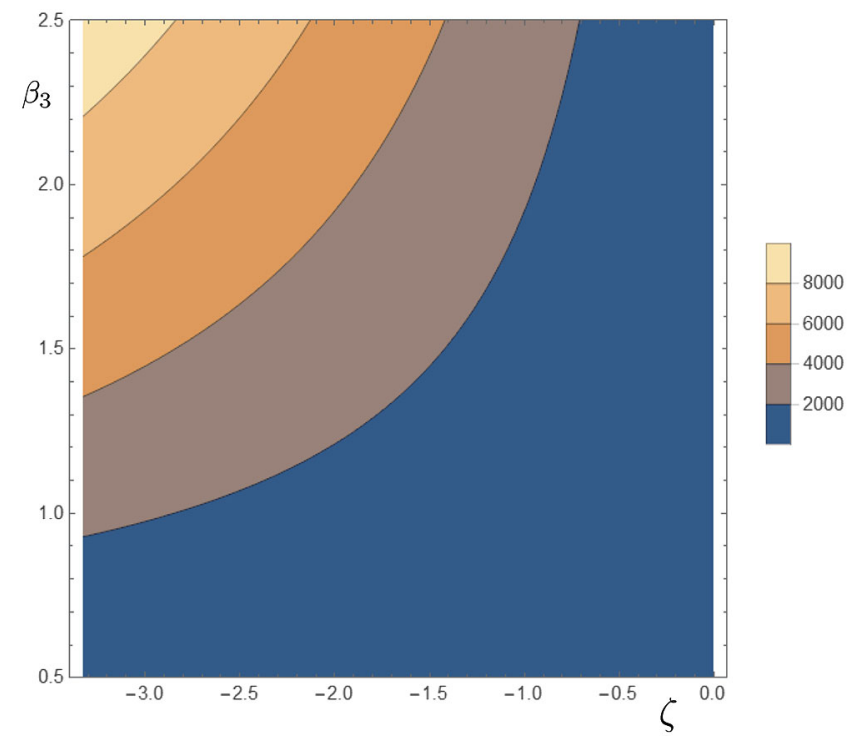

FIG. 16. The contour map of $E_{1} / E_{2}$ in terms of $\zeta$ and $\beta_{3}$. The dynamic range of $E_{1} / E_{2}$ is very wide from 1 to $10^{4}$.

is close to $\mathcal{S}_{\max }$, in the limit of $\zeta \rightarrow \zeta_{\min } \approx-3.326$ and $\beta_{3} \rightarrow \infty$.

However we find $E_{2}=0$ when $\alpha_{3}=0.5$. Since $E_{2} \geq 1$, we cannot choose $\alpha_{3}=0.5$. Hence choosing $\alpha_{3}=0.49999$ as well as $s_{2}=0.288675$, we show the contour maps of the efficiency $\eta$ and $E_{1} / E_{2}$ in Fig. 15 and 16.

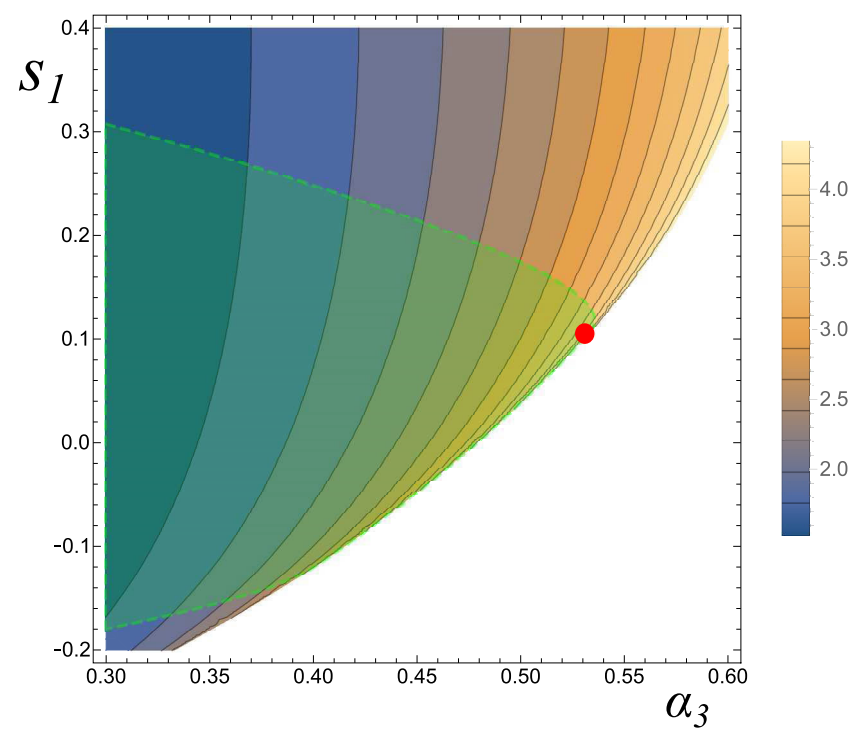

FIG. 17. The contour map of $E_{3}$ in terms of $\alpha_{3}$ and $s_{1}$ for the inverse Compton scattering in the case of $\sigma_{3}=1$. The timelike condition for the particle 3 is satisfied in the green shaded region. The maximal value $E_{3} \approx 4.202$ is found at the red point $\left(\alpha_{3}, s_{1}\right) \approx$ $(0.5331,0.1059)$.

The value of $E_{1} / E_{2}$ is larger than 1000 in the above range of parameters. Since $E_{2} \geq 1$, we find the efficiency is about 3.85 when $E_{1} \gtrsim 10^{3}$ [see Fig. 16]. If we take the limit of $\alpha_{3}=0.5$, the maximal efficiency is obtained $\eta_{\max }=$ $\mathcal{S}_{\max } \approx 3.876$ when $E_{1} \rightarrow \infty$.

The above result shows the efficiency can be larger than 3.5 but never exceeds $\mathcal{S}_{\max } \approx 3.876$ when the plunged photon energy is much larger than the particle 2 rest mass.

Note that we find the upper bound of the efficiency is about 3.732 even for the nonspinning case $\left(s_{2}=0\right)$.

\section{Case (C) MPM Inverse Compton scattering}

We first depict the contour map of $E_{3}$ in terms of $\alpha_{3}$ and $s_{1}$ in Fig. 17.

The maximal value $E_{3(\max )} \approx 4.202$ is obtained at the red point $\left(\alpha_{3}, s_{1}\right) \approx(0.5331,0.1059)$. From Eq. (3.24), we find the asymptotic behavior of $\mathcal{P}$ as

$$
\mathcal{P} \approx-8 E_{3} \zeta \beta_{3}\left[\frac{E_{3} g_{1}\left(s_{1}, \alpha_{3}\right)}{\left(1-s_{1}\right) f\left(s_{1}, E_{3}, \alpha_{3}\right)}+1\right],
$$

when we take a limit of $\zeta \beta_{3} \rightarrow-\infty$. Since $g_{1}\left(s_{1}, \alpha_{3}\right)>0$ in the plotted region of Fig. 17, we find $E_{2} \rightarrow 0$ as $\zeta \beta_{3} \rightarrow-\infty$. For the non-spinning particle 2 , only the condition $\zeta<0$ is required. As a result $\beta_{3}$ must be positive to get $E_{2} \rightarrow 0$. We obtain the maximal efficiency as $\eta_{\max }=$ $E_{3(\max )} \approx 4.202$. 
[1] S. Hawking, Phys. Rev. Lett. 26, 1344 (1971).

[2] R. Penrose, Riv. Nuovo Cimento 1, 252 (1969); Gen. Relativ. Gravit. 34, 1141 (2002).

[3] J. A. Wheeler, in Study Week on Nuclei of Galaxies, edited by D. J. K. O'Connell, Pontificiae Academiae Scientiarum Scripta Varia, No. 35 (North Holland, Amsterdam, 1971).

[4] J. M. Bardeen, W. H. Press, and S. A. Teukolsky, Astrophys. J. 178, 347 (1972).

[5] R. M. Wald, Astrophys. J. 191, 231 (1974).

[6] A. Kovetz and T. Piran, Lettere al Nuovo Cimento (1971-1985) 12, 39 (1975).

[7] Y. Zel'dovich, JETP Lett. 14, 180 (1971).

[8] Y. Zel'dovich, Sov. Phys. JETP 35, 1085 (1972).

[9] C. Misner, Phys. Rev. Lett. 28, 994 (1972).

[10] W. H. Press and S. A. Teukolsky, Nature (London) 238, 211 (1972).

[11] J. D. Bekenstein, Phys. Rev. D 7, 949 (1973).

[12] R. Brito, V. Cardoso, and P. Pani, Lect. Notes Phys. 906, 1 (2015).

[13] T. Piran, J. Shaham, and J. Katz, Astrophys. J. Lett. 196, L107 (1975).

[14] T. Piran and J. Shaham, Phys. Rev. D 16, 1615 (1977).

[15] M. Banados, J. Silk, and S. M. West, Phys. Rev. Lett. 103, 111102 (2009).

[16] T. Jacobson and T. P. Sotiriou, Phys. Rev. Lett. 104, 021101 (2010).

[17] E. Berti, V. Cardoso, L. Gualtieri, F. Pretorius, and U. Sperhake, Phys. Rev. Lett. 103, 239001 (2009).

[18] S.-W. Wei, Y.-X. Liu, H. Guo, and C.-E. Fu, Phys. Rev. D 82, 103005 (2010).

[19] M. Banados, B. Hassanain, J. Silk, and S. M. West, Phys. Rev. D 83, 023004 (2011).

[20] O. B. Zaslavskii, Phys. Rev. D 82, 083004 (2010).

[21] O. B. Zaslavskii, Pis'ma Zh. Eksp. Teor. Fiz. 92, 635 (2010); [JETP Lett. 92, 571 (2010)].

[22] A. A. Grib and Yu. V. Pavlov, Astropart. Phys. 34, 581 (2011).

[23] K. Lake, Phys. Rev. Lett. 104, 211102 (2010); 104, 259903 (E) (2010)

[24] T. Harada and M. Kimura, Phys. Rev. D 83, 024002 (2011).

[25] M. Kimura, K.-i. Nakao, and H. Tagoshi, Phys. Rev. D 83, 044013 (2011).

[26] M. Patil and P. S. Joshi, Phys. Rev. D 84, 104001 (2011).

[27] A. Abdujabbarov, N. Dadhich, B. Ahmedov, and H. Eshkuvatov, Phys. Rev. D 88, 084036 (2013).
[28] N. Tsukamoto, M. Kimura, and T. Harada, Phys. Rev. D 89, 024020 (2014).

[29] B. Toshmatov, A. Abdujabbarov, B. Ahmedov, and Z. Stuchlík, Astrophys. Space Sci. 357, 41 (2015).

[30] N. Tsukamoto and C. Bambi, Phys. Rev. D 91, 084013 (2015).

[31] C. Armaza, M. Bañados, and B. Koch, Classical Quantum Gravity 33, 105014 (2016).

[32] O. B. Zaslavskii, Europhys. Lett. 114, 30003 (2016).

[33] M. Guo and S. Gao, Phys. Rev. D 93, 084025 (2016).

[34] Y.-P. Zhang, B.-M. Gu, S.-W. Wei, J. Yang, and Y.-X. Liu, Phys. Rev. D 94, 124017 (2016).

[35] M. Bejger, T. Piran, M. Abramowicz, and F. Hakanson, Phys. Rev. Lett. 109, 121101 (2012).

[36] T. Harada, H. Nemoto, and U. Miyamoto, Phys. Rev. D 86, 024027 (2012); 86, 069902(E) (2012).

[37] J. D. Schnittman, Phys. Rev. Lett. 113, 261102 (2014).

[38] E. Leiderschneider and T. Piran, Phys. Rev. D 93, 043015 (2016).

[39] K. Ogasawara, T. Harada, and U. Miyamoto, Phys. Rev. D 93, 044054 (2016).

[40] O. B. Zaslavskii, Phys. Rev. D 94, 064048 (2016).

[41] E. Berti, R. Brito, and V. Cardoso, Phys. Rev. Lett. 114, 251103 (2015).

[42] M. Patil, T. Harada, K.-i. Nakao, P. S. Joshi, and M. Kimura, Phys. Rev. D 93, 104015 (2016).

[43] O. B. Zaslavskii, Phys. Rev. D 93, 024056 (2016).

[44] S. Mukherjee, Phys. Lett. B 778, 54 (2018).

[45] C. W. Misner, K. S. Thorne, and J. A. Wheeler, Gravitation (Freeman, San Francisco, 1971).

[46] A. Papapetrou, in Proc. R. Soc. A 209, 248 (1951).

[47] W. Dixon, Proc. R. Soc. A 314, 499 (1970).

[48] W. Dixon, Proc. R. Soc. A 319, 509 (1970).

[49] W. Dixon and J. Ehlers, in Proceedings of the International School of Physics Enrico Fermi, Course LXVII, edited by J. Ehlers (North Holland, Amsterdam, 1979), p. 156.

[50] M. Saijo, K.-i. Maeda, M. Shibata, and Y. Mino, Phys. Rev. D 58, 064005 (1998).

[51] K. S. Thorne, Astrophys. J. 191, 507 (1974).

[52] Y. Liu and W.-B. Liu, Phys. Rev. D 97, 064024 (2018).

[53] K.-i. Nakao, H. Okawa, and K.-i. Maeda, Prog. Theor. Exp. Phys. 2018, $013 E 01$ (2018).

[54] H. McAlister, T. A. ten Brummelaar, D. R. Gies, W. Huang, J. W. G. Bagnuolo, M. A. Shure, J. Sturmann, L. Sturmann, N. H. Turner, S. F. Taylor, D. H. Berger, E. K. Baines, E. Grundstrom, C. Ogden, S. T. Ridgway, and G. van Belle, Astrophys. J. 628, 439 (2005). 